\title{
The cooling of atomic and molecular gas in DR21 ${ }^{\star}$
}

\author{
H. Jakob ${ }^{1}$, C. Kramer ${ }^{1}$, R. Simon ${ }^{1}$, N. Schneider ${ }^{4,2,1}$, V. Ossenkopf ${ }^{1,3}$, S. Bontemps ${ }^{2}$, U. U. Graf ${ }^{1}$, and J. Stutzki ${ }^{1}$ \\ 1 KOSMA, I. Physikalisches Institut, Universität zu Köln, Zülpicher Straße 77, 50937 Köln, Germany \\ e-mail: jakob@ph1.uni-koeln.de \\ 2 Observatoire de Bordeaux, Université de Bordeaux 1, BP 89, 33270 Floirac, France \\ 3 SRON, National Institute for Space Research, PO Box 800, 9700 AV Groningen, The Netherlands \\ 4 SAp/CEA Saclay, 91191 Gif-sur-Yvette, France
}

Received 19 June 2006 / Accepted 11 September 2006

ABSTRACT

\begin{abstract}
Aims. We present an overview of a high-mass star formation region through the major (sub-)mm, and far-infrared cooling lines to gain insight into the physical conditions and the energy budget of the molecular cloud.

Methods. We used the KOSMA 3 m telescope to map the core $\left(10^{\prime} \times 14^{\prime}\right)$ of the Galactic star-forming region DR21/DR21 (OH) in the Cygnus $\mathrm{X}$ region in the two fine structure lines of atomic carbon $\left(\mathrm{CI}{ }^{3} \mathrm{P}_{1}-{ }^{3} \mathrm{P}_{0}\right.$ and $\left.{ }^{3} \mathrm{P}_{2}-{ }^{3} \mathrm{P}_{1}\right)$, in four mid- $J$ transitions of $\mathrm{CO}$ and ${ }^{13} \mathrm{CO}$, and in CS $J=7-6$. These observations were combined with FCRAO $J=1-0$ observations of ${ }^{13} \mathrm{CO}$ and $\mathrm{C}^{18} \mathrm{O}$. Five positions, including DR21, DR21 (OH), and DR21 FIR1, were observed with the ISO/LWS grating spectrometer in the [O I] 63 and $145 \mu \mathrm{m}$ lines, the [C II] $158 \mu \mathrm{m}$ line, and four high- $J$ CO lines. We discuss the intensities and line ratios at these positions and apply the local thermal equilibrium (LTE) and non-LTE analysis methods in order to derive physical parameters such as mass, density and temperature. The CO line emission was modeled up to $J=20$.

Results. From non-LTE modeling of the low- to high- $J$ CO lines, we identify two gas components, a cold one at temperatures of $T_{\text {kin }} \sim 30-40 \mathrm{~K}$ and one with $T_{\text {kin }} \sim 80-150 \mathrm{~K}$ at a local clump density of about $n\left(\mathrm{H}_{2}\right) \sim 10^{4}-10^{6} \mathrm{~cm}^{-3}$. While the cold quiescent component is massive, typically containing more than $94 \%$ of the mass, the warm, dense, and turbulent gas is dominated by mid- and high- $J$ CO line emission and its large line widths. The medium must be clumpy with a volume-filling of a few percent. The CO lines are found to be important in cooling the cold molecular gas, e.g. at DR21 (OH). Near the outflow of the UV-heated source DR21, the gas cooling is dominated by line emission of atomic oxygen and of CO. Atomic and ionised carbon play a minor role.
\end{abstract}

Key words. ISM: clouds - ISM: abundances - radio lines: ISM - line: profiles - stars: formation - ISM: individual objects: DR21 ISM: individual objects: DR21 $(\mathrm{OH})$

\section{Introduction}

Emission lines of $\mathrm{CII}, \mathrm{CI}$, and $\mathrm{CO}$ in molecular clouds are among the most important diagnostic probes of star formation and are particularly important for studying the influence of massive stars and their UV radiation on the environment. Obtaining the physical parameters of such regions is often done with simplified radiative transfer assumptions. Local thermal equilibrium (LTE) and the large velocity gradient (LVG) analysis have been proven to yield good results for the bulk of the cold gas (see, e.g., Black 2000). However, the assumption of a homogeneous medium does not represent the typical physical conditions of a high-mass star-formation region. Instead, the observing beam will contain several gas components with different excitation conditions and with unknown filling factors. The observed line transitions are sensitive to gas at different densities, temperatures, and opacities, corresponding to different regions. By selecting designated lines as tracers of the different parts of the gas, one can obtain a rather complete coverage of excitation conditions accounting for different physical and also chemical conditions within the beam. A more differenciated analysis using non-LTE radiative-transfer algorithms (see van Zadelhoff et al. 2002 , for an overview) is needed for this purpose. It can provide a more detailed description of the structure of the cloud

^ Appendices A-C are only available in electronic form at http: //www . aanda.org including clumpiness and temperature or density gradients (e.g., Ossenkopf et al. 2001; Williams et al. 2004).

Using the results of this analysis, we can try to retrieve a selfconsistent picture including all major processes driving and being driven by high-mass star-formation (e.g., with respect to turbulence or chemistry), thereby obtaining a detailed view of the photon-dominated regions (PDRs) in the environment of young stars. These PDRs are transition regions from ionised and atomic to dense molecular gas in which the physics and chemistry of the gas are dominated by the UV radiation from young massive stars. Processes heating and cooling the clouds are qualitatively well-understood on a theoretical level (e.g., Tielens \& Hollenbach 1985; Sternberg \& Dalgarno 1989), and in general we find a reasonable agreement between the theoretical predictions and observations of the brightest cooling lines. Still, there is an ongoing debate about the details of the energy input contributing to the heating. We find a combination of mechanisms, such as stellar far-ultraviolet radiation (FUV), dynamical energy through shocks or supernovae, or an enhanced cosmic-ray rate. There are also many open questions with respect to the chemical composition of these regions. The role of nonequilibrium chemistry is still not understood well. For instance, polycyclic aromatic hydrocarbons (PAHs) as a reservoir of fresh gas-phase carbon (e.g., Oka et al. 2004; Habart et al. 2005) may change the overall picture.

Emission from warm carbon in the form of atoms or ions or of carbonated molecules (mainly $\mathrm{CO}$ ) provides a significant 
fraction of the gas cooling at sub-millimeter and far-infrared (FIR) wavelengths (e.g., Giannini et al. 2000, 2001; Schneider et al. 2003; Kramer et al. 2004; Bradford et al. 2003, 2005). Finding the prevailing cooling channels among these and other species (e.g., [O I] $, \mathrm{H}_{2} \mathrm{O}, \mathrm{OH}$ ) may provide significant insight into the details of the chemical composition and the energy balance of PDRs. Since systematic observations of sub-mm and FIR cooling lines with good spatial resolution are still sparse, data to test the model predictions are still largely missing, and many questions concerning the details of the chemistry and energy balance remain unanswered. By studying a well-known, massive Galactic star-forming region, we aim at gaining new insight into these problems.

\section{The DR21 region}

The DR21 H II-region/molecular cloud is part of the Cygnus X complex of molecular clouds. Cygnus $\mathrm{X}$ is known to host a large number of H II-regions (Downes \& Rinehart 1966; Wendker 1984) associated with molecular clouds seen, as in the CfA ${ }^{12}$ CO $1-0$ survey (Leung \& Thaddeus 1992). An extended (11 square degrees) map of the Cygnus $\mathrm{X}$ region in the ${ }^{13} \mathrm{CO} 2-1$ line and smaller maps in the $\mathrm{CO}$ and ${ }^{13} \mathrm{CO} 3-2$ lines were obtained with the KOSMA telescope and are presented in Schneider et al. (2006) (from now on SBS2006). The distance to DR21 was sometimes estimated to be $\sim 3 \mathrm{kpc}$, based on visual interstellar extinction and radial velocity (e.g., Campbell et al. 1982). For this paper, we follow the argumentation in SBS2006 based on a morphological comparison of the molecular line data with mid-IR emission and the $\mathrm{OB}$ associations, and favour a value of $1.7 \mathrm{kpc}$ as the distance toward DR21 and DR21 $(\mathrm{OH})$. However, for easier comparison with the literature, we explicitly give all relevant physical properties as a function of distance.

The DR21 region has been subject to numerous studies at different wavelengths. Several active star-formation sites and cometary-shaped H II-regions were identified in maps of radio continuum emission (e.g., Dickel et al. 1983; Cyganowski et al. 2003). Ammonia observations by Mauersberger et al. (1985) were interpreted as evidence of high densities $\left(\sim 10^{5.5} \mathrm{~cm}^{-3}\right)$ paired with a high degree of clumpiness. The $\mathrm{H}_{2} \mathrm{O}$ masers (Genzel \& Downes 1977) and a recently obtained map of 1.3-mm continuum emission (Motte et al. 2005) show that DR21 belongs to a north-south orientated chain of massive starforming complexes. An additional indication of active star formation is also given by observations of vibrationally excited $\mathrm{H}_{2}$, tracing the hot, shocked gas in the vicinity of DR21 and DR21 (OH) (e.g., Garden et al. 1986; and more recent results by Davis et al. 2006). The $\mathrm{H}_{2}$ images reveal an extended $\left(5^{\prime}=\right.$ $2.5 \mathrm{pc}(D / 1.7 \mathrm{kpc}))$ northeast to southwest orientated outflow centred on DR21. To the west, the outflow is highly collimated, whereas toward the eastern side, gas is expanding in a blister style (Lane et al. 1990). Jaffe et al. (1989) found very intense CO 7-6 emission peaking on the H II-region and following the $\mathrm{H}_{2}$-emission.

In this paper, we present the results of sub-mm mapping observations with KOSMA and discuss far-IR line intensities observed with ISO. This comprehensive data set allowed us to study the spatial and, partly also, the kinematic structure of all major cooling lines of the ISM in the vicinity of DR21 and DR21(OH). In Sect. 3, we analyse the line emission and continuum data toward five positions, by comparing the spatial distribution, line profiles, and the physical properties through selected line ratios and the far-IR dust continuum. In Sect. 4, we model the integrated line intensity ratios and absolute intensities with radiative transfer models. The impact of the line emission on the total cloud cooling is dicussed in Sect. 5, and a summary is given in Sect. 6.

\section{Observations}

We used the KOSMA-3 m submm-telescope to map the $\mathrm{DR} 21 / \mathrm{DR} 21(\mathrm{OH})$ region in seven rotational mid- $J$ transitions of $\mathrm{CO},{ }^{13} \mathrm{CO}$, and $\mathrm{CS}$ and in the two fine-structure transitions of $\mathrm{C}$ I. These data were combined with ISO/LWS FIR line observations toward single positions of [O I], [C II], and $\mathrm{CO}$. The dataset is complemented with ${ }^{13} \mathrm{CO}$ and $\mathrm{C}^{18} \mathrm{O} 1-0$ FCRAO observations.

\subsection{KOSMA}

The region centred on DR21/DR21 $(\mathrm{OH})$ was mapped in the two atomic carbon fine-structure transitions at $492 \mathrm{GHz}(609 \mu \mathrm{m}$, ${ }^{3} \mathrm{P}_{1}-{ }^{3} \mathrm{P}_{0}$; hereafter $\left.1-0\right)$ and $809 \mathrm{GHz}\left(370 \mu \mathrm{m},{ }^{3} \mathrm{P}_{2}-{ }^{3} \mathrm{P}_{1}\right.$; hereafter $2-1)$ and the mid- $J$ rotational transitions of $\mathrm{CO}(J=3-2$, $J=4-3, J=6-5$, and $J=7-6),{ }^{13} \mathrm{CO}(J=3-2$, and $J=6-5$ ) using the dual channel 345 and $230 / 660 \mathrm{GHz}$ SIS receiver, and the SubMillimeter Array Receiver for Two frequencies (SMART) at the KOSMA telescope. SMART is a dualfrequency, eight-pixel SIS-heterodyne receiver that simultaneously observes in the 650 and $350 \mu \mathrm{m}$ atmospheric windows (Graf et al. 2002). All observations were taken in the period between 2003 and 2006.

An area of $\sim 10^{\prime} \times 14^{\prime}$ was mapped in the two [C I] lines and the CO 7-6 line on a $27.5^{\prime \prime} \times 27.5^{\prime \prime}$ grid with a total integration time of $160 \mathrm{~s}$ per position. The observations before 2005 were performed in DBS, with a secondary mirror chop throw of 6 ' fixed in azimuth. Due to the extent of the DR21 cloud complex, self-chopping is present in some of the DBS-mode observations. In Appendix B, we describe how we processed these data in order to reduce the impact of the self-chopping. The $\mathrm{CO}$ and ${ }^{13} \mathrm{CO} 3-2$, and $\mathrm{CO} 4-3$ were observed in an OTF mapping mode on a fully-sampled grid. A zeroth-order baseline was subtracted from the calibrated spectra. In addition to $\mathrm{CO} 3-2$, we simultaneously obtained the CS 7-6 line in the image sideband of the $345 \mathrm{GHz}$ channel. Pointing was frequently checked on Jupiter, the sun, and then confirmed on DR21 itself. The resulting accuracy was better than $\pm 20^{\prime \prime}$. Larger ${ }^{13} \mathrm{CO} 3-2$ and 2-1 maps are presented in SBS2006. Due to their lower spatial resolution, we did not consider the $J=2-1$ data here.

The ${ }^{12} \mathrm{CO}$ and ${ }^{13} \mathrm{CO} 6-5$ observations were presented in Köster (1998). The typical DSB receiver temperatures in the high-frequency channel was between $160-220 \mathrm{~K}$. The ${ }^{13} \mathrm{CO} 6-5$ observations cover a region of $\sim 3^{\prime} \times 3^{\prime}$ centred on DR21, while the ${ }^{12} \mathrm{CO}$ observations extend farther to the north covering a region of $\sim 3^{\prime} \times 6^{\prime}$.

Atmospheric calibration was done by measuring the atmospheric emission at the reference position to derive the opacity. Sideband imbalances were corrected using standard atmospheric models (Cernicharo 1985) and assuming a receiver sideband gain of 0.5. Spectra taken simultaneously in the two frequency bands near 492 and $810 \mathrm{GHz}$ were calibrated with an averaged water-vapor value. The [C I] 2-1 and CO 7-6 lines, observed simultaneously in two sidebands, were also corrected for sideband imbalance. Their pointing is identical, and the relative calibration error between these two transitions is very small. We scaled the antenna temperature data to the $T_{\mathrm{mb}}$ scale using main beam efficiencies $\eta_{\mathrm{eff}}$ as listed in Table 1 . The HPBWs (40" 
Table 1. List of observational parameters for KOSMA.

\begin{tabular}{|c|c|c|c|c|c|c|c|c|c|c|}
\hline Species & Transition & $\begin{array}{c}\text { Frequency } \\
{[\mathrm{GHz}]}\end{array}$ & Receiver & $\eta_{\mathrm{mb}}^{a}$ & $\begin{array}{c}\mathrm{HPBW}^{a} \\
{\left[{ }^{\prime \prime}\right]}\end{array}$ & Mode & 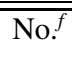 & $\begin{array}{c}\Delta v^{f} \\
{\left[\mathrm{~m} \mathrm{~s}^{-1}\right]}\end{array}$ & 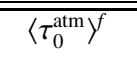 & Observing period \\
\hline${ }^{13} \mathrm{CO}^{b}$ & $J=3-2$ & 330.5880 & DualSIS & 0.68 & 80 & OTF $^{c}$ & 693 & 308 & 0.26 & $01 / 2006$ \\
\hline${ }^{12} \mathrm{C}^{32} \mathrm{~S}$ & $J=7-6$ & 342.8829 & & 0.68 & 80 & $\mathrm{OTF}^{c}$ & 525 & 294 & 0.08 & $12 / 2005$ \\
\hline${ }^{12} \mathrm{CO}$ & $J=3-2$ & 345.7960 & & 0.68 & 80 & $\mathrm{OTF}^{c}$ & 525 & 294 & 0.08 & $12 / 2005$ \\
\hline${ }^{12} \mathrm{CO}$ & $J=4-3$ & 461.0408 & SMART & 0.5 & 57 & $\mathrm{OTF}^{c}$ & 3458 & 677 & $0.85-1.4$ & $1 / 2005$ \\
\hline$\left[{ }^{12} \mathrm{C} \mathrm{I}\right]$ & ${ }^{3} \mathrm{P}_{1}-{ }^{3} \mathrm{P}_{0}$ & 492.1607 & & 0.5 & 55 & $\mathrm{DBS}^{d}$ & 1380 & 630 & $0.5-1.6$ & $1 / 2003-3 / 2004$ \\
\hline${ }^{13} \mathrm{CO}$ & $J=6-5$ & 661.067 & & 0.4 & $\sim 40$ & $\mathrm{PSw}^{e}$ & 33 & 307 & $1.0-1.3$ & $1 / 1998$ \\
\hline${ }^{12} \mathrm{CO}$ & $J=6-5$ & 691.473 & & 0.4 & $\sim 40$ & $\mathrm{PSw}^{e}$ & 58 & 295 & $1.0-1.3$ & $1 / 1998$ \\
\hline${ }^{12} \mathrm{CO}$ & $J=7-6$ & 806.6518 & & 0.31 & 42 & $\mathrm{DBS}^{d}$ & 964 & 775 & $0.4-1.6$ & $1 / 2003-3 / 2004$ \\
\hline$\left[{ }^{12} \mathrm{CI}\right]$ & ${ }^{3} \mathrm{P}_{2}-{ }^{3} \mathrm{P}_{1}$ & 809.3420 & & 0.31 & 42 & $\mathrm{DBS}^{d}$ & 964 & 775 & $0.4-1.6$ & $1 / 2003-3 / 2004$ \\
\hline
\end{tabular}

${ }^{a}$ The main beam efficiency $\eta_{\mathrm{mb}}$ and the half power beam width (HPBW) were determined from cross scans of Jupiter. ${ }^{b}$ See Schneider et al. (2006) for a ${ }^{13} \mathrm{CO} 3-2$ map covering a larger part of Cygnus X. ${ }^{c}$ OTF - on-the-fly mapping mode with periodic switching to an emission-free reference position at $\alpha(\mathrm{J} 2000)=20^{\mathrm{h}} 37^{\mathrm{m}} 10^{\mathrm{s}}, \delta(\mathrm{J} 2000)=42^{\circ} 30^{\prime} 00^{\prime \prime} .{ }^{d} \mathrm{DBS}-$ dual beam switch observing mode with a chopping secondary mirror in azimuth. ${ }^{e}$ The $\mathrm{CO}$ and ${ }^{13} \mathrm{CO} 6-5$ observations were carried out on a $35^{\prime \prime}$ grid (Köster 1998) in a position-switched observing mode (PSw). The OFF position was at $\alpha(J 2000)=20^{\mathrm{h}} 26^{\mathrm{m}} 43^{\mathrm{s}} .56, \delta(J 2000)=42^{\circ} 09^{\prime} 39^{\prime} \cdot{ }^{\prime} .^{f}$ These columns lists the number of mapped positions, the velocity channel width $\Delta v$ (the resolution is a factor of $\sim 1.4$ larger), and the mean atmospheric zenith opacity $\left\langle\tau_{0}^{\text {atm }}\right\rangle$.
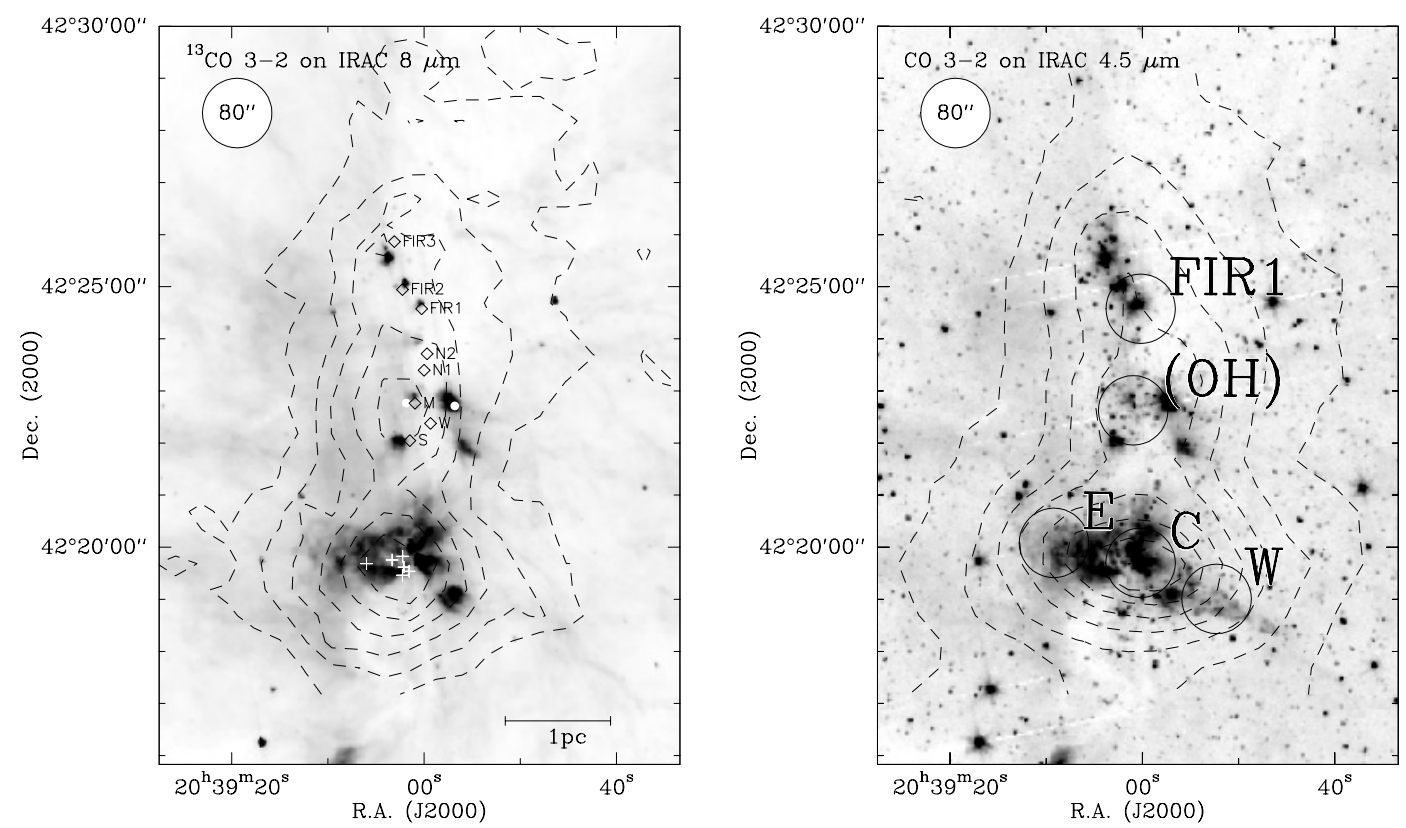

Fig. 1. View of the DR21/DR21 $(\mathrm{OH})$ high-mass star-forming region. Left: overlay of the KOSMA ${ }^{13} \mathrm{CO} 3-2$ map (cf. Schneider et al. 2006/SBS2006) in contours on a Spitzer IRAC $8 \mu \mathrm{m}$ greyscale map (Marston et al. 2004). Contours are on a $T_{\mathrm{mb}}$ scale and range from 16.8 to 100.8 in steps of $16.8 \mathrm{~K} \mathrm{~km} \mathrm{~s}^{-1}\left(\sigma=5.6 \mathrm{~K} \mathrm{~km} \mathrm{~s}^{-1}\right)$. The integration interval is -10 to $20 \mathrm{~km} \mathrm{~s}^{-1}$. The left-top circle indicates the HPBW of KOSMA. The map also shows 6 positions of radio continuum sources identified as OB stars by Roelfsema et al. (1989) (white crosses), 2 infrared sources and maser positions (Garden et al. 1986) (small white circles), and 8 compact 1.3-mm dust emission sources (Chandler et al. 1993) (open rectangles). Right: overlay of the Spitzer map at $4.5 \mu \mathrm{m}$ with contours of the KOSMA ${ }^{12} \mathrm{CO} 3-2$ map (integration interval of -20 to $30 \mathrm{~km} \mathrm{~s}^{-1}$; discussed in SBS2006) ranging from 18 to 450 in steps of $48 \mathrm{~K} \mathrm{~km} \mathrm{~s}^{-1}\left(\sigma=6 \mathrm{~K} \mathrm{~km} \mathrm{~s}^{-1}\right)$. The labeled circles mark the positions and beam sizes of the ISO/LWS observations that we used to constrain the physical parameters as discussed in the text. The LWS beam is comparable to the resolution of the KOSMA maps.

to $80^{\prime \prime}$ ) and $\eta_{\mathrm{mb}}$ were determined using continuum scans on Jupiter. From observations of standard calibration sources, we estimate that the absolute calibration is accurate to within $\sim 15 \%$. Most spectra were taken under excellent weather conditions with typical values of the atmospheric opacity along the line-of-sight better than 1.0 at 350 and $600 \mu \mathrm{m}$, and better than 0.5 above $870 \mu \mathrm{m}$.

\subsection{ISO LWS observations and data reduction}

The ISO long wavelength spectrometer (LWS, Clegg et al. 1996) 43-197 $\mu \mathrm{m}$ grating scans (AOT L01) were obtained for
5 positions in the DR21 region from the ISO Data Archive (IDA) (see Table 2 and Fig. 7). We removed duplicate scans, overlapping positions, or data with a very poor spectral resolution from the sample.

The grating scans contain the FIR continuum, the atomic fine structure lines of [O I] at 63 and $145 \mu \mathrm{m}$, ionised atomic lines of [C II] at $158 \mu \mathrm{m},[\mathrm{O}$ III] at 52 and $88 \mu \mathrm{m},[\mathrm{N} \mathrm{II}]$ at $122 \mu \mathrm{m}$, and the high- $J$ CO molecular lines $J=14-13$ through $J=17-16$. All spectra were processed with the ISO Spectral Analysis Package (ISAP, v. 2.1). In ISAP, the data were deglitched by hand, defringed (detectors 4-9), and corrected for flux clipping (the extended source correction). Some artefacts remain after the 

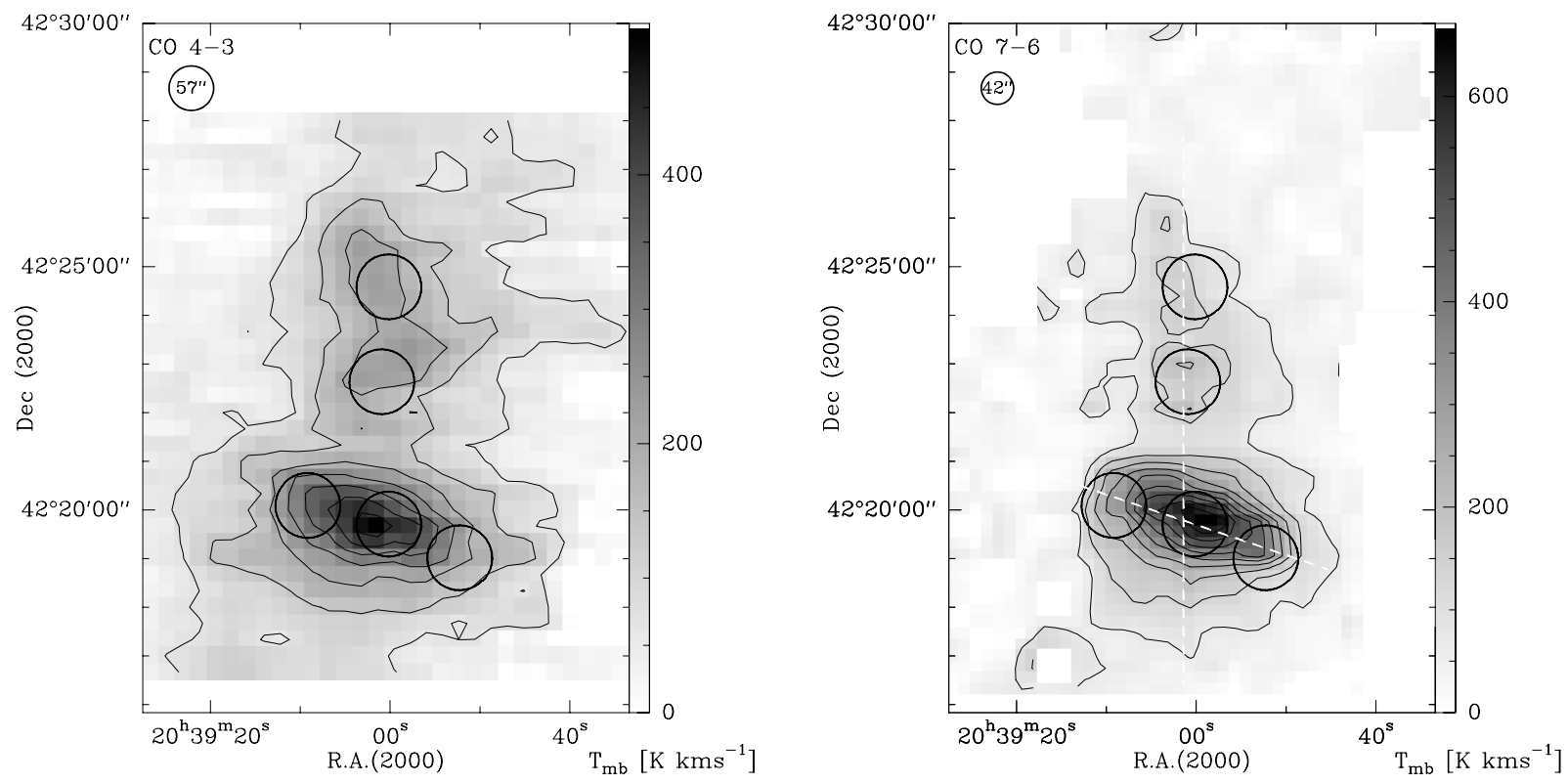

Fig. 2. Integrated intensity map of CO 4-3 (left) and CO 7-6 (right) of DR21 and DR21(OH). The integration interval is -20 to $30 \mathrm{~km} \mathrm{~s}^{-1}$. The contours for CO $4-3$ run from $66(3 \sigma)$ to $462 \mathrm{~K} \mathrm{~km} \mathrm{~s}^{-1}$ in steps of $3 \sigma$, and from $68(2 \sigma)$ to $671 \mathrm{~K} \mathrm{~km} \mathrm{~s}^{-1}$ in steps of $2 \sigma$ for CO $7-6$. The HPBW is indicated in the upper left corner. The positions of ISO/LWS observations (cf. Fig. 1) are indicated by circles. The dashed white lines correspond to the cuts shown in Fig. 6.
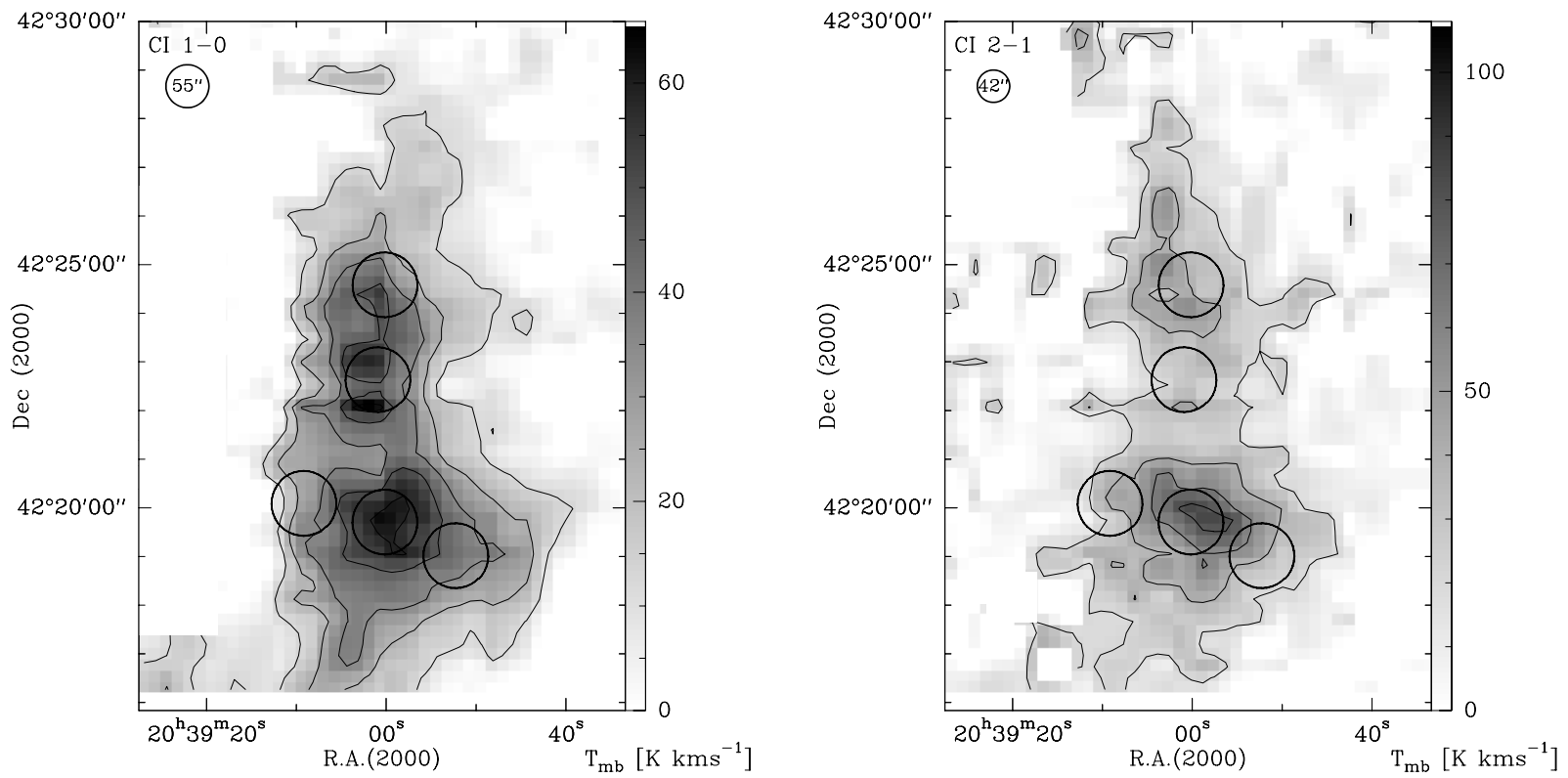

Fig. 3. Integrated intensity map of [C I] 1-0 (left) and of [C I $] 2-1$ (right). The integration interval is -10 to $20 \mathrm{~km} \mathrm{~s}^{-1}$. The contours for C I $1-0$ run from $12(2 \sigma)$ to $60 \mathrm{~K} \mathrm{~km} \mathrm{~s}^{-1}$ in steps of $2 \sigma$, and from $20(1 \sigma)$ to $108 \mathrm{~K} \mathrm{~km} \mathrm{~s}^{-1}$ in steps of $\sigma$ for $[\mathrm{CI}] 2-1$.

Table 2. Positions of ISO observations in absolute coordinates.

\begin{tabular}{lcll}
\hline \hline Name $^{*}$ & TDT-No. & RA [J2000] & Dec [J2000] \\
\hline DR21 E/East & 15200785 & $20^{\mathrm{h}} 39^{\mathrm{m}} 09^{\mathrm{s}} .14$ & $42^{\circ} 20^{\prime} 05^{\prime \prime} .0$ \\
DR21 C/Center & 15200786 & $20^{\mathrm{h}} 39^{\mathrm{m}} 00^{\mathrm{s}} .93$ & $42^{\circ} 19^{\prime} 42^{\prime \prime} 0$ \\
DR21 W/West & 15200787 & $20^{\mathrm{h}} 38^{\mathrm{m}} 52^{\mathrm{s}} .23$ & $42^{\circ} 19^{\prime} 00^{\prime} .5$ \\
DR21 (OH) & 34700439 & $20^{\mathrm{h}} 39^{\mathrm{m}} 00^{\mathrm{s}} .95$ & $42^{\circ} 22^{\prime} 37^{\prime \prime} 6$ \\
DR21 FIR1 & 35500317 & $20^{\mathrm{h}} 39^{\mathrm{m}} 00^{\mathrm{s}} .2$ & $42^{\circ} 24^{\prime} 34^{\prime \prime} 6$ \\
\hline
\end{tabular}

* DR21 C is centred on the HiI-region. The projected distance of DR21 E (resp. DR21 W) from position DR21 C is $0.77 \mathrm{pc}$ (resp. $0.89 \mathrm{pc}$ ) at a distance of $1.7 \mathrm{kpc}$.

processing (see Fig. 7) and were not corrected. Line strengths were measured within ISAP. See Table 4 for further details.

\subsection{FCRAO}

We used ${ }^{13} \mathrm{CO}$ and $\mathrm{C}^{18} \mathrm{O} 1-0$ data from a large-scale mapping project of the whole Cygnus X region using the FCRAO $14 \mathrm{~m}$ telescope. These data were obtained between December 2003 and January 2006 and will be presented in more detail in upcoming papers (Simon et al., in prep.). The data were observed using the 32 pixel array SEQUOIA in an OTF mapping mode (at an angular resolution of $\left.\sim 50^{\prime \prime}\right)$. In this paper, we use smoothed data to have the same angular resolution of $80^{\prime \prime}$ as the KOSMA data.

\section{Results}

Figure 1 gives an overview centred on DR21 (OH) (designated as $\mathrm{W} 75 \mathrm{~S}$ by some authors). The Spitzer $8 \mu \mathrm{m}$ and $4.5 \mu \mathrm{m}$ data 
by Marston et al. (2004) show the small-scale structure of this region at angular resolutions between $1.3^{\prime \prime}-3^{\prime \prime}$. Several bright IR objects, in particular near DR2 $1 \mathrm{C}^{1}$, delineate the dense ridge of gas and dust in both wavelength bands. The map of $8 \mu \mathrm{m}$ emission is dominated by many streamers of emission, which appear to connect to the ridge, and diffuse emission. This is mainly emission from polycyclic aromatic hydrocarbons (PAHs), which are the prime absorbers of far-ultraviolet (FUV) radiation from the surrounding and embedded massive stars. Bright $8 \mu \mathrm{m}$ emission therefore highlights the photon-dominated surface regions of molecular clouds (PDRs). The molecular outflow along DR21 E, C, and W is traced well by $4.5 \mu \mathrm{m}$ emission, mainly due to strong lines of shock-excited or UV-pumped $\mathrm{H}_{2}$ $v=0-0 \mathrm{~S}(9)$. No ro-vibrational lines of $\mathrm{CO} v=1-0$ and only weak $\operatorname{Br} \alpha$ have been found in this band (Smith et al. 2006).

\subsection{Line-integrated maps}

\subsection{1. $\mathrm{CO}$ and ${ }^{13} \mathrm{CO}$ maps}

Figure 1 (contours on right panel) and Fig. 2 show the KOSMA maps of integrated emission of the rotational transitions $\mathrm{CO} 3-2$, 4-3, and 7-6, which preferentially trace the warm and dense molecular gas around DR21 and along the north-south orientated molecular ridge. Apart from the ridge, the most obvious feature in the maps is the line emission along the outflow axis oriented NE-SW. The main axis of the strong 3-2, 4-3, and 7-6 emission $\left(F W H M \sim 230^{\prime \prime}\right.$, resp. $\left.\sim 1.9 \mathrm{pc}(D / 1.7 \mathrm{kpc})\right)$ is tilted by $20^{\circ}$ with respect to the right ascension axis and correlates nicely with extended $\mathrm{H}_{2}$ emission lobes (Garden et al. 1986). The red-shifted $\mathrm{CO}$ line-wing emission is stronger toward the west side, whereas the blue-shifted emission is more pronounced to the east indicating that this side is closer to the observer.

Morphologically, the CO 3-2 map shows a very similar shape in comparison to the CO 4-3 data. Detailed differences can be seen better if the maps are smoothed to the same resolution. In Fig. 6, we present two cuts along the outflow and along the main ridge from these data. At DR21, all CO line integrated intensities are very similar. Toward position DR21 E, the emission of CO 3-2 and 4-3 stays at a high level, while the 7-6 emission drops. Toward the west, all three line intensities decline in a similar fashion. Away from DR21, along the $\mathrm{N}-\mathrm{S}$ cut, the $\mathrm{CO} 3-2$ emission is dominant, whereas $\mathrm{CO} 4-3$ and CO 7-6 are sequentially weaker. Strong CO 7-6 emission is limited to the DR2 1 core. DR2 $1(\mathrm{OH})$ is located $180^{\prime \prime}$ north of DR21 and clearly separated from the DR21 region by a gap in the ridge. DR2 $1(\mathrm{OH})$ is bright in the lower $J$ transitions, but appears weaker in the $J=7-6$ transition.

${ }^{13} \mathrm{CO} 3-2$ (contours on left panel of Fig. 1) was selected to map the column density in the region. This transition traces the molecular ridge showing two maxima of emission at DR21 C and DR21 (OH). The total mass of the ridge is $3.5 \times 10^{4} M_{\odot}(D / 1.7 \mathrm{kpc})^{2}$ (SBS2006). ${ }^{13} \mathrm{CO}$ weakly follows the outflow from DR21 E to W. Following the molecular ridge farther to the north, an elongated emission band roughly coincides in position with three far-infrared sources. The southernmost source, DR21 FIR1, was observed by ISO/LWS. Marston et al. (2004) find an extremly red object (ERO 3) here, illuminating a shell-like halo in the $4.5 \mu \mathrm{m}$ band. The other two, FIR2 and FIR3, coincide with MSX point sources. Like the FIR sources, this northern region is slightly tilted to the east, indicating flux

${ }^{1}$ We use the ISO/LWS position names throughout the paper (cf. Table 2).

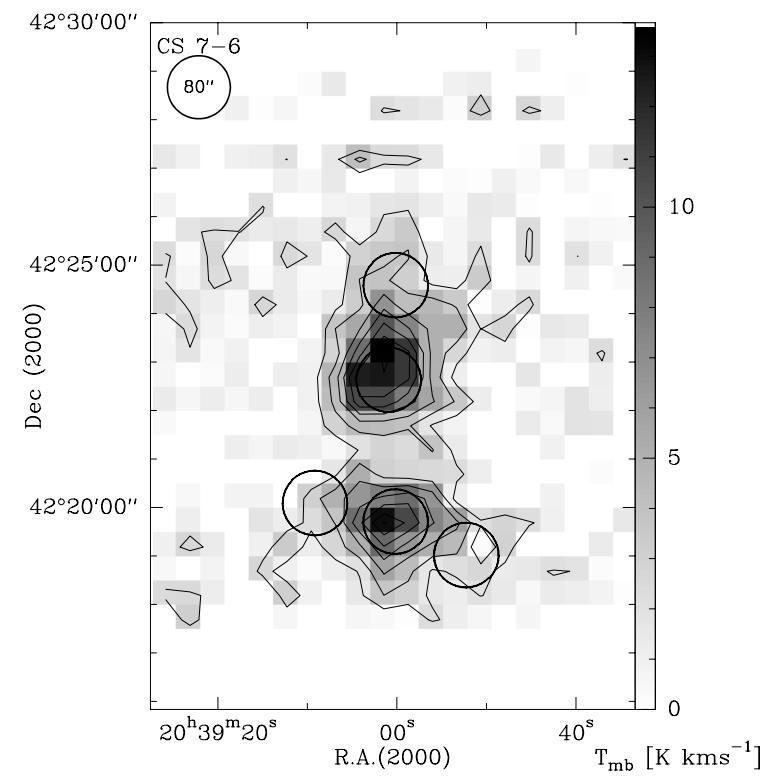

Fig. 4. Integrated intensity map of CS 7-6. The integration interval is -10 to $4 \mathrm{~km} \mathrm{~s}^{-1}$. Contours run from $1.8(3 \sigma)$ to $13.0 \mathrm{~K} \mathrm{~km} \mathrm{~s}^{-1}$ (in steps of $3 \sigma)$.

contribution to mainly low- $J \mathrm{CO}$ emission from all three embedded far-IR sources. ${ }^{13} \mathrm{CO}$ reaches up to FIR3, which marks the edge of the dense ridge to the north.

\subsection{2. [CI] maps}

C I 1-0 and 2-1 appear in good agreement with the shape of the molecular ridge as traced by ${ }^{13} \mathrm{CO}$. Even though the emission around DR21 in [CI] (see Fig. 3) appears more centrally peaked than in the $\mathrm{CO}$ maps, the main axis between positions $\mathrm{E}$ and $\mathrm{W}$ is clearly visible. The molecular ridge is seen to extend from the southeast through DR21 and through DR21 $(\mathrm{OH})$ $3^{\prime}$ northwards. DR21 $(\mathrm{OH})$ is visible as an elongated, less compact region linking-up to the far-infrared sources farther north. The DR21 $(\mathrm{OH})$ region appears to harbour multiple sources, as there is no central emission peak. The [C I] 1-0 map reveals a southern peak and extended weaker emission in the north. This substructure may not be significant because the variation is only about $2 \sigma$. Chandler et al. (1993) identified five compact sources in the DR21 $(\mathrm{OH})$ region (indicated in Fig. 1) using 1.3-mm thermal dust emission at $11^{\prime \prime}$ resolution. The C I 2-1 is, compared to $1-0$, relatively weak toward DR21 $(\mathrm{OH})$ and suggests a lower temperature than for DR21 C (see below). North of DR21 (OH), the carbon emission stretches over the three far-IR spots FIR1, FIR2, and FIR3. Here, the upper atomic carbon line is in good agreement with the overall shape of optically thin low- $J$ CO isotopomers, i.e., the $\mathrm{C}^{18} \mathrm{O} 2-1$ map by Wilson \& Mauersberger (1990), and to some extent with our ${ }^{13} \mathrm{CO} 3-2$ map. A more distinct morphology analysis is not possible due to the remaining noise and some self-chopping residuals.

\subsubsection{CS 7-6 map}

The map in Fig. 4 reveals two condensations at DR21 and around DR21 $(\mathrm{OH})$. The southern peak is centred on the H II-region and follows the direction of the outflow to the west. The FWHM is $133^{\prime \prime} \times 102^{\prime \prime}$. The northern complex $\left(\right.$ FWHM $\left.108^{\prime \prime} \times 156^{\prime \prime}\right)$ is extended and may contain several unresolved cores (see above). 


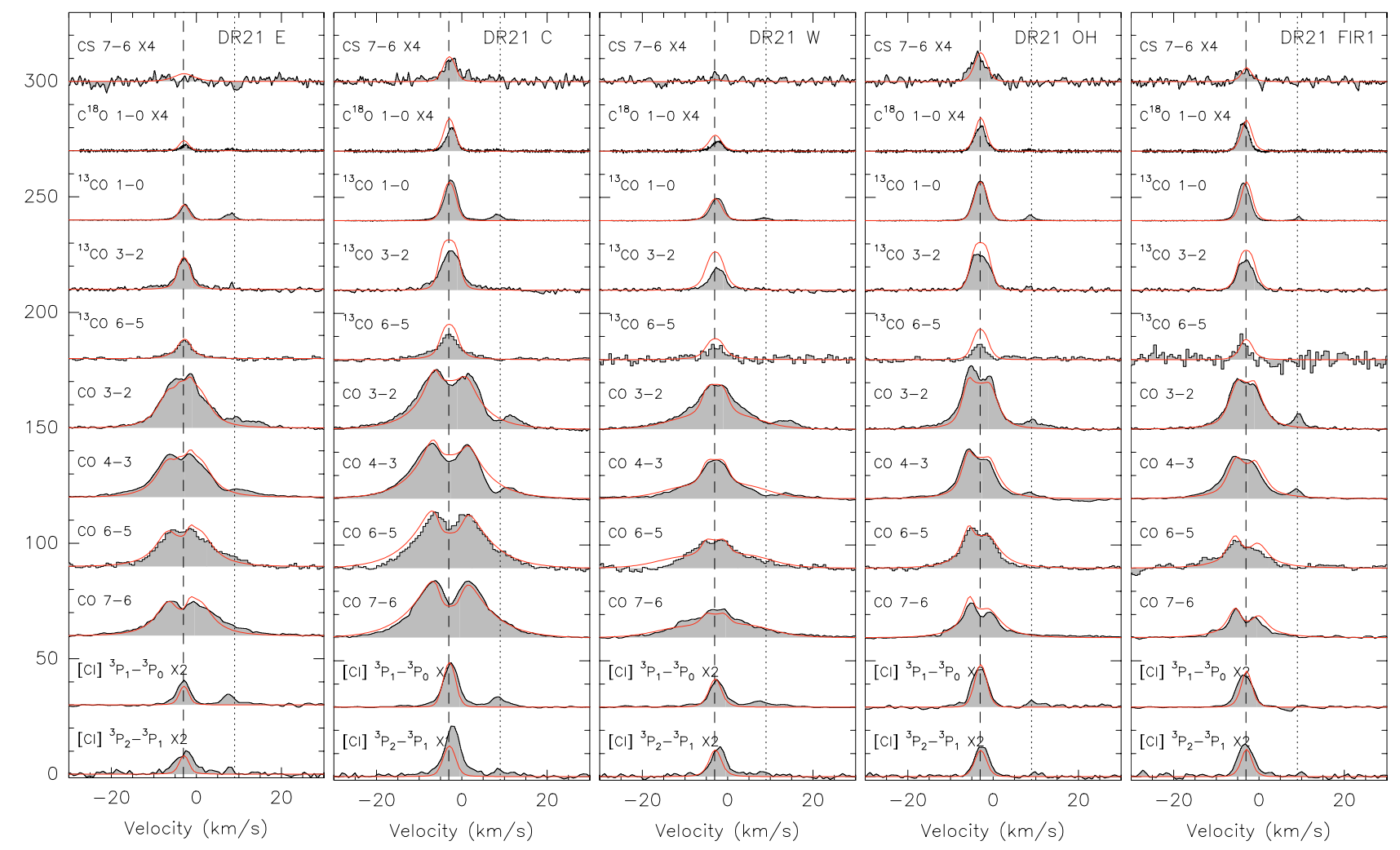

Fig. 5. Spectra of all observed species at the five ISO positions. Grey filled spectra outlined in black: observed lines; thin grey lines: line profiles modeled with the radiative transfer code SIMLINE (see Sect. 4.2). All spectra are shown at a common spatial resolution of $80^{\prime \prime}$ and on a $T_{\mathrm{mb}} \mathrm{scale}$. The baselines are shifted in steps of $30 \mathrm{~K}$. [C I] was multiplied by a factor of 2 , and CS 7-6 and $\mathrm{C}^{18} \mathrm{O} 1-0$ by a factor of 4 . The $J=1-0$ transition data are FCRAO observations. The dashed lines indicate the central velocity of $-3 \mathrm{~km} \mathrm{~s}^{-1}$, and the dotted lines the secondary velocity component at about $9 \mathrm{~km} \mathrm{~s}^{-1}$, which was excluded from the fitting.

The emission extends up to the FIR1 position, but does not cover all three FIR spots. The lines of CS $7-6$ and $\mathrm{C}^{18} \mathrm{O} 1-0$ are the only lines in our sample that show brighter emission toward DR21 $(\mathrm{OH})$ than toward the southern molecular cloud/H IIregion DR21. For CS, this has also been observed by Shirley et al. (2003).

\subsection{Line profiles}

In the following, we concentrate on the 5 ISO/LWS positions (as indicated in Fig. 1). In Fig. 5, we present spectra of ${ }^{12} \mathrm{CO}$, ${ }^{13} \mathrm{CO}$, and $\mathrm{C}^{18} \mathrm{O}$ towards these positions, together with spectra of C I, and of CS 7-6 between -30 to $30 \mathrm{~km} \mathrm{~s}^{-1}$. We present all observations at a common resolution of $80^{\prime \prime}$. In Table 3 , we give the corresponding line fluxes.

The ${ }^{12} \mathrm{CO}$ emission from the DR21/H II-region shows broad wing emission due to the outflowing molecular gas (with line widths of more than $15 \mathrm{~km} \mathrm{~s}^{-1}$ and peak temperatures well above $20 \mathrm{~K})$. A self-absorption dip at the velocity of $-3 \mathrm{~km} \mathrm{~s}^{-1}$ is most pronounced in mid- $J$ CO lines at DR2 $1 \mathrm{C}$ where the peak intensity drops by a factor of two at the line centre. Toward DR21 (OH), CO shows blue-skewed, double-peaked lines with self-absorption. In both cases, the velocity of the line reversal nicely matches the corresponding emission in ${ }^{13} \mathrm{CO}$ and $\mathrm{C} \mathrm{I}$ and is very likely caused by the same cold material in the dust lane.

With widths of 4 to $6 \mathrm{~km} \mathrm{~s}^{-1}$, the line shapes of ${ }^{13} \mathrm{CO}$ are similar to the $[\mathrm{CI}]$ profiles. The ${ }^{13} \mathrm{CO} 6-5$ emission $\left(E_{\mathrm{u}} / k_{\mathrm{B}}=\right.$ $79.3 \mathrm{~K})$ at all five positions indicates the presence of warm and dense gas. At DR2 $1 \mathrm{C},{ }^{13} \mathrm{CO} 6-5$ shows broad symmetric wing emission that is underlying a narrow component. The velocity extent of the wings is similar to that in mid- $J$ CO lines. A better signal-to-noise ratio would allow a more accurate estimate. Only weak line wings are visible in the lower ${ }^{13} \mathrm{CO} 3-2$ line since it primarily traces the quiescent gas.

DR21 is associated with molecular gas centred at a velocity of $-3 \mathrm{~km} \mathrm{~s}^{-1}$. In addition to this, weaker emission between $\sim 7$ and $14 \mathrm{~km} \mathrm{~s}^{-1}$ is associated with the $\mathrm{W} 75 \mathrm{~N}$ complex approximately $11 \mathrm{pc}(D / 1.7 \mathrm{kpc})$ to the northwest. Some authors (e.g., Dickel et al. 1978) conclude that both clouds are actually interacting. SBS2006 shows channel maps revealing a link between both clouds. Diffuse emission in the ${ }^{13} \mathrm{CO} J=1-0$ and both [CI] transitions and absorption in the red wing of the low- $J$ CO lines (up to $J=4-3$ ) suggest that this material is located in front of the DR21 complex. We do not discuss this contribution further here but instead refer the reader to SBS2006.

\subsection{ISO archive data}

Figure 7 shows the ISO/LWS continuum fluxes, together with spectrally unresolved lines of [C II], [O I], [O III], [N II], and rotational high- $J \mathrm{CO}$ transitons. The following sections discuss the dust and line emission at the individual positions.

\subsubsection{Grey body fit to the continuum}

In all five cases, the far-infrared dust-continuum flux - with strong emission lines masked out - was $\chi^{2}$-fitted with a single isothermal grey-body model $F\left(T_{\text {dust }}, \tau_{\text {dust }}\right)_{\lambda}=\Omega B_{\lambda}\left(T_{\text {dust }}\right)(1-$ $\left.\mathrm{e}^{-\tau_{\text {dust }}}\right)$ with a fixed solid angle $\left(\Omega=1.5 \times 10^{-7} \mathrm{sr}\right.$, assuming that the emission fills the $80^{\prime \prime}$ aperture) and a dust opacity 
Table 3. Line-integrated intensities in $\left[\mathrm{K} \mathrm{km} \mathrm{s}^{-1}\right]$ (upper table) and in $\left[10^{-7} \mathrm{erg} \mathrm{s}^{-1} \mathrm{~cm}^{-2} \mathrm{sr}^{-1}\right]$ (lower table) of the low- and mid- $J$ CO isotopomeric lines and of the CS 7-6 line observed at KOSMA and FCRAO. In order to prevent the $+9 \mathrm{~km} \mathrm{~s}^{-1}$ velocity component from adding to the integrated intensity, the listed intensities were integrated only over the $-3 \mathrm{~km} \mathrm{~s}^{-1}$ component. As the ${ }^{12} \mathrm{CO}$ lines merge with the second velocity component, these spectra were fitted using a 2-component Gaussian with the $+9 \mathrm{~km} \mathrm{~s}^{-1}$ velocity component masked out. All line intensities are reported for $80^{\prime \prime}$ angular resolution (higher angular resolution data have been smoothed).

\begin{tabular}{|c|c|c|c|c|c|c|c|c|c|c|c|}
\hline Position & $\overline{\mathrm{CO}}$ & $\overline{\mathrm{CO}}$ & $\overline{\mathrm{CO}}$ & $\overline{\mathrm{CO}}$ & ${ }^{13} \mathrm{CO}$ & ${ }^{13} \mathrm{CO}$ & ${ }^{13} \mathrm{CO}$ & $\overline{\mathrm{C}^{18} \mathrm{O}}$ & $\overline{[\mathrm{CI}]}$ & $\overline{\overline{[\mathrm{CI}}]}$ & $\overline{\mathrm{CS}}$ \\
\hline units in $\left[\mathrm{K} \mathrm{km} \mathrm{s}^{-1}\right]$ & $3-2$ & $4-3$ & $6-5$ & $7-6$ & $1-0$ & $3-2$ & $6-5$ & $1-0$ & $1-0$ & $2-1$ & $7-6$ \\
\hline DR21 E & 348.7 & 283.4 & 247.2 & 265.7 & 22.38 & 69.4 & 43.1 & 1.66 & 21.9 & 28.2 & $\overline{3.0}$ \\
\hline $\mathrm{DR} 21 \mathrm{C}$ & 440.0 & 462.6 & 434.4 & 477.5 & 70.48 & 112.2 & 80.9 & 8.26 & 46.6 & 58.4 & 13.8 \\
\hline DR21 W & 230.5 & 221.9 & 177.7 & 223.1 & 41.02 & 47.6 & 33.9 & 3.67 & 26.9 & 28.5 & 2.9 \\
\hline DR21 $(\mathrm{OH})$ & 283.2 & 197.4 & 162.0 & 153.2 & 67.92 & 91.5 & 24.9 & 9.03 & 39.5 & 30.2 & 13.6 \\
\hline DR21 FIR1 & 223.8 & 193.2 & 130.2 & 107.3 & 59.49 & 61.9 & 26.0 & 9.16 & 33.4 & 33.0 & 3.9 \\
\hline Position & $\mathrm{CO}$ & $\mathrm{CO}$ & $\mathrm{CO}$ & $\mathrm{CO}$ & ${ }^{13} \mathrm{CO}$ & ${ }^{13} \mathrm{CO}$ & ${ }^{13} \mathrm{CO}$ & $\mathrm{C}^{18} \mathrm{O}$ & {$[\mathrm{CI}]$} & $\overline{[\mathrm{C} \mathrm{I}]}$ & $\mathrm{CS}$ \\
\hline in $\left[10^{-7} \mathrm{erg} \mathrm{s}^{-1} \mathrm{~cm}^{-2} \mathrm{sr}^{-1}\right]$ & $3-2$ & $4-3$ & $6-5$ & $7-6$ & $1-0$ & $3-2$ & $6-5$ & $1-0$ & $1-0$ & $2-1$ & $7-6$ \\
\hline DR21E & 147.8 & 284.6 & 837.6 & 1429.1 & 0.307 & 25.7 & 127.6 & 0.023 & 26.8 & 153.4 & $\overline{1.3}$ \\
\hline DR21 C* & 186.5 & 464.6 & 1471.9 & 2568.6 & 0.967 & 41.5 & 239.8 & 0.112 & 56.9 & 317.3 & 5.7 \\
\hline DR21 W & 97.7 & 222.9 & 602.1 & 1200.2 & 0.563 & 17.6 & 100.3 & 0.050 & 32.5 & 154.7 & 1.2 \\
\hline DR21 $(\mathrm{OH})$ & 120.0 & 198.3 & 548.9 & 824.1 & 0.932 & 33.9 & 73.8 & 0.122 & 48.3 & 164.7 & 5.6 \\
\hline DR21 FIR1 & 94.8 & 194.0 & 441.2 & 577.0 & 0.816 & 22.9 & 77.0 & 0.124 & 40.8 & 179.5 & 1.6 \\
\hline
\end{tabular}

* Boreiko \& Betz (1991) give CO $\left({ }^{13} \mathrm{CO}\right) 9-8$ intensities of $2.3 \pm 0.08 \times 10^{-4}\left(0.45 \pm 0.1 \times 10^{-4}\right) \mathrm{erg} \mathrm{s}^{-1} \mathrm{~cm}^{-2} \mathrm{sr}^{-1}$ for a $80^{\prime \prime}\left(83^{\prime \prime}\right)$ beam and a telescope coupling efficiency of 0.6 .

$\tau_{\text {dust }} \sim \tau_{\mathrm{v}} \lambda^{-\beta}$ with $\beta=1.5$. Spectral indices are typically found between 1.0 and 2.0 (see Goldsmith et al. 1997, for a discussion of observations and grain models). Although the spectral index is known to vary with the cloud type and optical depth, we assumed a fixed $\beta$ for all 5 positions (but see discussion in Sect. 4.2.3). A fixed $\beta$ of 1.5 is compatible with the grain models of Preibisch et al. (1993), Pollack et al. (1994), and observations, e.g., by Walker et al. (1990). The total infrared dust continuum flux and bolometric luminosity per position was derived by integrating the fit-model $F_{\lambda}$. The integration interval $(1-1000 \mu \mathrm{m})$ covers the expected intensity maxima between $50-80 \mu \mathrm{m}$ and corresponds to the range used, e.g., by Calzetti et al. (2000). Errors in the fit parameters were estimated as described in the following. We assumed (i) the optical depths $\tau_{\text {dust }}$ and total fluxes are accurate to about $\sim 10 \%$, mainly due to the remaining calibration artefacts within the $L W 1-5$ bands; (ii) the temperature fit error is less than $2 \%$, but the absolute error also depends on the quality of SW1 detector data (leftmost data points in Fig. 7).

We found extinctions between $20^{\mathrm{m}} \leq A_{\mathrm{v}} \leq 260^{\mathrm{m}}$ and temperatures between $31-43 \mathrm{~K}$ (cf. Table 5). In contrast to the extinction, the variation in dust temperature is small. Considering that we used a single-temperature model, these temperatures were probably biased by the warmer dust. Evidence of cold dust peaking above $100 \mu \mathrm{m}$ is visible at DR2 $1 \mathrm{~W}, \mathrm{E},(\mathrm{OH})$ and FIR1 in Fig. 7; but disentangling this component would require longer wavelength data, and the contribution to the integrated greybody flux is relatively small. The cold dust is studied in detail by, e.g., Chandler et al. (1993) and Motte et al. (2005). We derived the molecular hydrogen column density from the conversion $N\left(\mathrm{H}_{2}\right) / A_{\mathrm{v}} \approx 0.94 \times 10^{21} \mathrm{~cm}^{-2} \mathrm{mag}^{-1}$ (Bohlin et al. 1978, cf. Table 5), and if placed at the distance of $1.7 \mathrm{kpc}$, the molecular gas masses vary between $150-1800 M_{\odot}$.

The peak of the beam-averaged dust temperature is $\sim 43 \mathrm{~K}$ at DR21 C. With $A_{\mathrm{v}}>100^{\mathrm{m}}$, the strongest continuum sources are DR21 C and DR21(OH). Due to the high temperature towards DR2 $1 \mathrm{C}$, the flux density is higher at short wavelengths, but drops below the flux density of DR21(OH) above $\sim 200 \mu \mathrm{m}$. For DR21 (OH), we estimated a higher mass than for the DR21 E-W complex (1814, resp. $\left.1257 M_{\odot}\right)$. Chandler et al. (1993) assumed temperatures between $25 \mathrm{~K}$ and $40 \mathrm{~K}$ and derived a slightly lower total mass of $1650 M_{\odot}(D / 1.7 \mathrm{kpc})^{2}$ for the sources at DR21 $(\mathrm{OH})$.

\subsubsection{ISO/LWS far-IR lines: carbon monoxide and ionised carbon}

The LWS-band covers many important far-IR cooling lines and tracers of the photon-dominated regime of molecular clouds. Table 4 gives the integrated line intensities of [C II] $(158 \mu \mathrm{m})$, high- $J$ CO, and [OI] (63 and $145 \mu \mathrm{m})$ derived from the ISO observations quoted above. In this paragraph, we focus on the carbon-bearing species needed for Sect. 4 and postpone the discussion of the other lines to the end of Sect. 4.

At DR21, highly-excited CO emission is visible at all three positions, but most prominently around the H II-region at DR21C. The centre position with more than $2.4 \times$ $10^{-4} \mathrm{erg} \mathrm{s}^{-1} \mathrm{~cm}^{-2} \mathrm{sr}^{-1}$ in mid- and high- $J \mathrm{CO}$ is also the brightest continuum source in the mapped region. The emission stems from large columns of warm quiescent gas that are heated by an embedded object. Toward the East and West positions, where vibrationally excited $\mathrm{H}_{2}$ outlines the molecular outflow (Garden et al. 1986), the FIR continuum is somewhat weaker and more important, the continuum flux does not correlate with the unusually strong FIR line emission. This gas is visible in the line wings of mid- $J$ CO lines (Fig. 5) and in the bright $\mathrm{H}_{2}$ line emission at $4.5 \mu \mathrm{m}$ in the IRAC map (Fig. 1). Lane et al. (1990) conclude that neither a single $\mathrm{C}$ - nor a single J-shock can account for the observed far-IR line emission alone and additional heating through FUV radiation is required. Although DR21 $(\mathrm{OH})$ has the second strongest continuum, the sources associated with this position are surprisingly inactive in far-IR line emission. We find four high- $J$ CO line detections, but due to a lower scanning resolution, the values given in Table 4 have a higher uncertainty. The far-IR source FIR1 is the faintest with respect to the line fluxes, although the continuum is stronger than towards DR $21 \mathrm{~W}$. High$J$ CO was not detected at FIR1, so we give upper limits in the table.

The [C II] emission is detected at all five positions and peaks at DR21 E. Toward the West, the flux decreases by almost $50 \%$. The low [C II] flux of $2.3 \times 10^{-4} \mathrm{erg} \mathrm{s}^{-1} \mathrm{~cm}^{-2} \mathrm{sr}^{-1}$ at DR21 (OH) 
Table 4. The ISO/LWS high- $J$ CO and atomic FIR lines at $80^{\prime \prime}$ resolution. The transformation between flux densities and surface brightnesses was made using the beam sizes given in Table 5.10 of the ISO-LWS Handbook v. 2.1. The beam solid angle varies between $8 \times 10^{-8} \mathrm{sr}$ and $1.4 \times 10^{-7} \mathrm{sr}$. Because the lines are unresolved, the line widths were held to the instrumental line width: 0.29 or $0.60 \mu \mathrm{m}$ for detectors $0-4$ and 5-9, respectively. Correction factors between 0.37 to 0.85 for extended sources have been applied. We assume a $30 \%$ absolute photometry uncertainty for LWS.

\begin{tabular}{lllllllrrrr}
\hline \hline Position & CO & CO & CO & CO & [C II $]$ & [O I $]$ & {$[\mathrm{OI}]$} & {$[\mathrm{O}$ III $]$} & {$[\mathrm{O}$ III] } & [N II $]^{a}$ \\
in $\left[10^{-4} \mathrm{erg} \mathrm{s}^{-1} \mathrm{~cm}^{-2} \mathrm{sr}^{-1}\right]$ & $14-13$ & $15-14$ & $16-15$ & $17-16$ & $158 \mu \mathrm{m}$ & $63 \mu \mathrm{m}$ & $145 \mu \mathrm{m}$ & $52 \mu \mathrm{m}$ & $88 \mu \mathrm{m}$ & $122 \mu \mathrm{m}$ \\
\hline DR21 E & 0.25 & 0.72 & 0.51 & 0.33 & 8.2 & 48.2 & 2.9 & 3.53 & 3.08 & 0.435 \\
DR21 C & 1.75 & 2.46 & 0.99 & 0.63 & 8.1 & 49.4 & 6.15 & 11.5 & 4.64 & $<0.75$ \\
DR21 W & 0.44 & 0.69 & 0.42 & 0.29 & 4.7 & 10.1 & 1.136 & 1.78 & 2.52 & 0.306 \\
DR21 (OH) & $(0.45)$ & $(0.99)$ & $(0.40)$ & $(0.50)$ & 2.3 & 2.94 & 0.845 & $(1.27)$ & 1.56 & $<2.7$ \\
DR21 FIR1 $^{c}$ & $<0.28$ & $<0.3$ & $<0.28$ & $<0.25$ & 3.6 & 1.78 & 0.45 & 0.54 & 1.66 & $<0.6$ \\
\hline
\end{tabular}

${ }^{a}\left[\mathrm{~N}\right.$ II] was clearly found only toward the outflow positions E and W. At the other positions, we give $3 \sigma$ upper limits. ${ }^{b}$ For DR21 (OH), numbers in parentheses are more uncertain $(S / N$ between $1-3)$ due to a poorer frequency resolution. ${ }^{c}$ At DR21 FIR1, no detection of CO was possible, so we give $3 \sigma$ upper limits.

Table 5. Results of dust continuum single-component fit, with $\beta$ fixed at 1.5 , and $\Omega$ is $1.5 \times 10^{-7} \mathrm{sr}$.

\begin{tabular}{lccccccc}
\hline \hline Position & $\tau_{\mathrm{v}}$ & $A_{\mathrm{v}}(\mathrm{ISO})^{a}$ & $\begin{array}{c}N\left(\mathrm{H}_{2}\right) \\
{\left[10^{22} \mathrm{~cm}^{-2}\right]}\end{array}$ & $\begin{array}{c}\text { Mass } \\
{\left[M_{\odot}(D / 1.7 \mathrm{kpc})^{2}\right]}\end{array}$ & $\begin{array}{c}T_{\text {dust }} \\
{[\mathrm{K}]}\end{array}$ & $\begin{array}{c}I_{\mathrm{IR}}{ }^{b} \\
{\left[\mathrm{erg} \mathrm{s}^{-1} \mathrm{~cm}^{-2} \mathrm{sr}^{-1}\right]}\end{array}$ & $\left.\begin{array}{c}L \\
{\left[10^{3}\right.}\end{array} L_{\odot}(D / 1.7 \mathrm{kpc})^{2}\right]$ \\
\hline DR21 E & 20 & $21.2 \pm 1.9$ & 2.1 & 148 & $41.6 \pm 0.6$ & $0.55 \pm 0.05$ & 7 \\
DR21 C & 112 & $121 \pm 11$ & 12.1 & 850 & $42.5 \pm 0.7$ & $3.4 \pm 0.3$ & 45 \\
DR21 W & 34 & $36.9 \pm 3.5$ & 3.69 & 259 & $30.7 \pm 0.4$ & $0.18 \pm 0.02$ & 2 \\
DR21 (OH) & 238 & $259 \pm 43$ & 25.8 & 1814 & $31.2 \pm 0.8$ & $1.3 \pm 0.2$ & 17 \\
DR21 FIR1 & 45 & $48.4 \pm 4.3$ & 4.84 & 340 & $33.3 \pm 0.5$ & $0.37 \pm 0.03$ & 5 \\
\hline
\end{tabular}

${ }^{a}$ The extinction and the opacity are linked through $A_{\mathrm{v}}=2.5 \tau_{\mathrm{v}} \cdot \log _{10} e \approx 1.085 \cdot \tau_{\mathrm{v}} \cdot{ }^{b}$ The total infrared flux is calculated from $I_{\mathrm{IR}}=\int F_{\lambda} \mathrm{d} \lambda$.
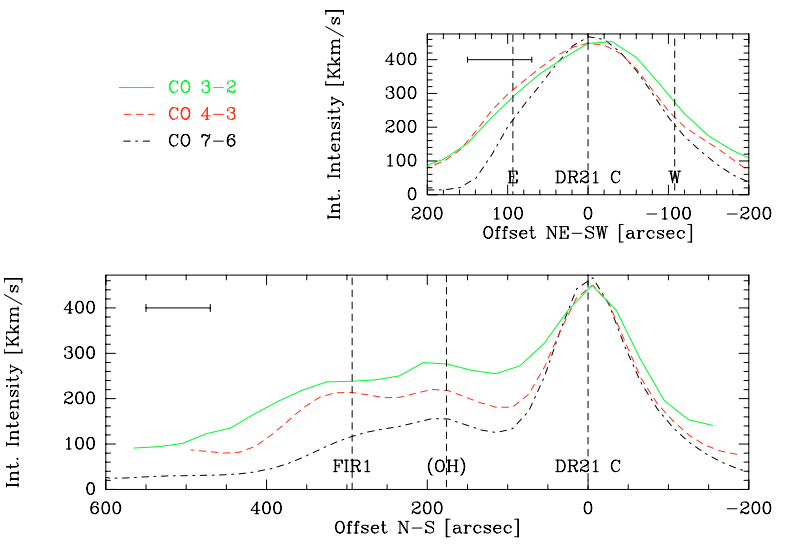

Fig. 6. Cut along the DR21 outflow axis (top) and along the ridge (bottom) centred on DR21 C in the CO 3-2, 4-3, and 7-6 integrated emission. The $80^{\prime \prime}$ resolution is indicated as a horizontal line. The dashed vertical lines indicate the positions of the ISO/LWS observations.

may indicate a lack of ionised carbon or that the temperature is too low for excitation in the upper energy level $\left(E_{\mathrm{u}} / k_{\mathrm{B}}=91 \mathrm{~K}\right.$ above ground). At DR21 FIR1, [C II] is again slightly enhanced compared to DR21 $(\mathrm{OH})$.

\section{The physical structure of DR21}

In this section we analyse the line properties of the observed carbon species from ISO/LWS data and combine these results with the KOSMA observations. We start with an LTE approach and then model the line emission with full radiative transfer.

\subsection{Comparison of $\mathrm{CO}, \mathrm{C}$, and $\mathrm{C}^{+}$}

We estimated the composition of the molecular gas with respect to the carbon abundance in the main gas-phase species $\mathrm{CO}, \mathrm{C}$,

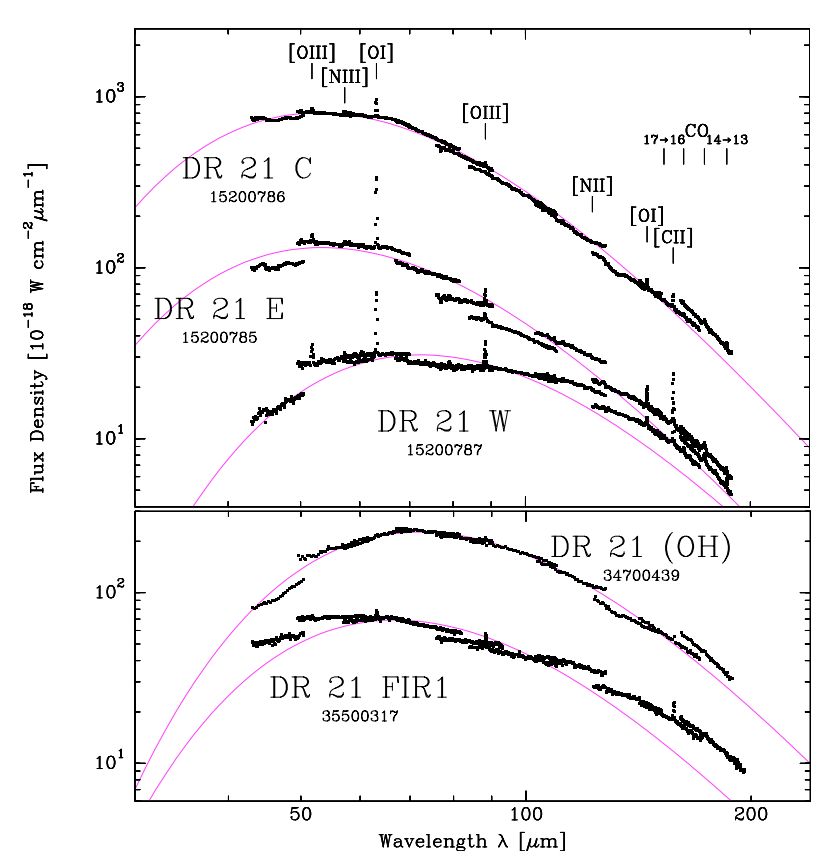

Fig. 7. ISO/LWS Spectral Energy Distribution (SED) of the DR21 region (upper panel), and the DR21 $(\mathrm{OH})$ and DR21 FIR1 region (lower panel). Curves indicate the best greybody fit corresponding to the values given in Table 5 .

and $\mathrm{C}^{+}$. This was done by assuming LTE and optically thin line emission. We therefore used the less abundant isotopomeres ${ }^{13} \mathrm{CO}$ and $\mathrm{C}^{18} \mathrm{O}$. We list the results for excitation temperature, column density, and abundance in Table 6. 
Table 6. C I and ${ }^{13} \mathrm{CO}$ line ratio $R$ (in $\mathrm{K} \mathrm{km} \mathrm{s}^{-1}$; values taken from Table 3), excitation temperature (with lower and upper $1 \sigma$ limits), and $\mathrm{CI}$, ${ }^{13} \mathrm{CO}$ (with relative upper and lower errors), and $\mathrm{C}^{18} \mathrm{O}$ column densities (bracketed by $T_{\text {ex }}$ of ${ }^{13} \mathrm{CO}$ ). All abundances $X$ are relative to the $\mathrm{H}_{2}$ column densities (first row; taken from Col. 4 of Table 5).

\begin{tabular}{|c|c|c|c|c|c|c|}
\hline Line/lineratio & Source property & $\overline{\mathrm{DR} 21 \mathrm{E}}$ & $\overline{\mathrm{DR} 21 \mathrm{C}}$ & $\overline{\mathrm{DR} 21 \mathrm{~W}}$ & $\overline{\text { DR21 }(\mathrm{OH})}$ & 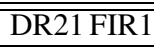 \\
\hline $\mathrm{H}_{2 \text {,dust }}$ & $N\left(\mathrm{H}_{2}\right)\left[10^{22} \mathrm{~cm}^{-2}\right]$ & 2.1 & 12.1 & 3.69 & 25.8 & 4.84 \\
\hline \multirow[t]{4}{*}{$\mathrm{CI}_{\mathrm{I}}$} & $R_{\mathrm{C}_{\text {I }}}\left(\mathrm{C}_{\text {I }} 2-1 / 1-0\right)$ & $1.29 \pm 0.36$ & $1.25 \pm 0.35$ & $1.06 \pm 0.30$ & $0.77 \pm 0.22$ & $0.99 \pm 0.28$ \\
\hline & $T_{\mathrm{ex},[\mathrm{C} I]}[\mathrm{K}]$ & $79_{47}^{157}$ & $74_{50}^{140}$ & $56_{41}^{88}$ & $38_{31}^{51}$ & $51_{39}^{76}$ \\
\hline & $N(\mathrm{C}$ I $)\left[10^{17} \mathrm{~cm}^{-2}\right]$ & $3.2_{-0.2}^{+0.3}$ & $6.8_{-0.4}^{+0.6}$ & $3.8_{-0.2}^{+0.3}$ & $5.2_{-0.1}^{+0.2}$ & $4.6_{-0.2}^{+0.3}$ \\
\hline & $X(\mathrm{C} \mathrm{I})\left[10^{-5}\right]$ & 1.5 & 0.56 & 1.03 & 0.20 & 0.10 \\
\hline \multirow[t]{3}{*}{${ }^{13} \mathrm{CO} 6-5 / 3-2$} & $R_{13 \mathrm{CO}, \text { high }}\left({ }^{13} \mathrm{CO} 6-5 / 3-2\right)$ & $0.62 \pm 0.26$ & $0.72 \pm 0.31$ & $0.71 \pm 0.30$ & $0.27 \pm 0.12$ & $0.42 \pm 0.18$ \\
\hline & $T_{\text {ex, }{ }^{13} \mathrm{CO}, \text { high }}[\mathrm{K}]$ & $43_{36}^{53}$ & $46_{38}^{58}$ & $46_{38}^{58}$ & $30_{26}^{34}$ & $35_{30}^{41}$ \\
\hline & $N\left({ }^{13} \mathrm{CO} 6-5\right)\left[10^{15} \mathrm{~cm}^{-2}\right]$ & $33.0_{+11.1}^{-9.0}$ & $53.8_{+20.4}^{-12.8}$ & $22.6_{+8.5}^{-5.4}$ & $39.6_{+21.3}^{-10.7}$ & $27.9_{+13.4}^{-7.2}$ \\
\hline \multirow[t]{3}{*}{${ }^{13} \mathrm{CO} 3-2 / 1-0$} & $R_{13} \mathrm{CO}$,low $\left({ }^{13} \mathrm{CO} 3-2 / 1-0\right)$ & $3.10 \pm 1.30$ & $1.59 \pm 0.67$ & $1.16 \pm 0.49$ & 1.35. \pm 0.57 & $1.04 \pm 0.44$ \\
\hline & $T_{\mathrm{ex},{ }^{13} \mathrm{CO}, \text { low }}[\mathrm{K}]$ & $25_{16}^{37}$ & $15_{12}^{19}$ & $13_{10}^{16}$ & $14_{11}^{17}$ & $12_{10}^{15}$ \\
\hline & $N\left({ }^{13} \mathrm{CO} 1-0\right)\left[10^{15} \mathrm{~cm}^{-2}\right]$ & $33.4_{-8.9}^{+12.3}$ & $75.6_{-8.6}^{+12.2}$ & $40.0_{-4.6}^{+5.1}$ & $69.1_{-80}^{11.6}$ & $56.5_{-4.3}^{+7.2}$ \\
\hline \multirow[t]{2}{*}{$\mathrm{C}^{18} \mathrm{O} 1-0$} & $N\left(\mathrm{C}^{18} \mathrm{O}\right)\left[10^{15} \mathrm{~cm}^{-2}\right]$ & $2.4-3.8$ & $8.7-20.0$ & $3.5-8.9$ & $9.0-15.1$ & $8.5-17.7$ \\
\hline & $X\left(\mathrm{C}^{18} \mathrm{O}\right)\left[10^{-7}\right]$ & $1.2-1.8$ & $0.72-1.7$ & $0.95-2.4$ & $0.35-0.59$ & $1.7-3.7$ \\
\hline \multirow[t]{3}{*}{ C II } & $N\left(\left[\mathrm{C}_{\mathrm{II}}\right]\right)^{a}\left[10^{17} \mathrm{~cm}^{-2}\right]$ & 5.25 & 5.18 & 3.00 & 1.47 & 2.30 \\
\hline & $X(\mathrm{C}$ II $)\left[10^{-5}\right]$ & 2.5 & 0.43 & 0.81 & 0.057 & 0.48 \\
\hline & {$\left[\mathrm{C}^{+}\right]:\left[\mathrm{C}^{0}\right]:[\mathrm{CO}]^{b}[\%]$} & $26: 16: 58$ & $10: 12: 78$ & $16: 12: 72$ & 03:10:87 & 05:09:85 \\
\hline
\end{tabular}

${ }^{a}$ The C II column density was estimated using a method described in Sect. 4.1. ${ }^{b}$ This row lists the relative abundance of carbon in the three major gas phase species $\mathrm{C}$ II, C I, and $\mathrm{CO}$. The $\mathrm{CO}$ column density is derived from the lower $\mathrm{C}^{18} \mathrm{O}$ column density assuming a relative $[\mathrm{CO}]:\left[\mathrm{C}^{18} \mathrm{O}\right]$ abundance of 490.

\subsubsection{Atomic carbon}

The C I excitation temperature needed to produce the observed line ratios can be estimated since both $\mathrm{C}$ I lines are likely to be optically thin (cf. line profiles in Fig. 5). The line temperature ratio $R_{\mathrm{C}_{\mathrm{I}}}$ falls between 0.8 at $\mathrm{DR} 21(\mathrm{OH})$ and 1.3 close to and east of DR21. The temperature is determined from this ratio using $T_{\mathrm{ex}}=38.8 \mathrm{~K} / \ln \left(2.11 / R_{\mathrm{CI}}\right)(\mathrm{Eq}$. (C.5)). The range $(38 \mathrm{~K}$ at $(\mathrm{OH})$ to $79 \mathrm{~K}$ at $\mathrm{E})$ indicates the origin of the atomic carbon lines in a warm environment. Zmuidzinas et al. (1988) derived a temperature of $32 \mathrm{~K}$ from the two $\mathrm{C}$ I lines at DR2 $1(\mathrm{OH})$ that is close to our value at this position. Our results for $T_{\mathrm{ex}}$ are lower compared to other Galactic high-mass star-forming regions (e.g., W3 Main and S 106 with typically $T_{\mathrm{ex}} \geq 110 \mathrm{~K}$, Kramer et al. 2004; Schneider et al. 2003), but substantially higher compared to the average temperature in the inner Galactic disk of $\sim 20 \mathrm{~K}$ (Fixsen et al. 1999). The beam-averaged column density, 3.2-6.8 $\times 10^{17} \mathrm{~cm}^{-2}$ (Eq. (C.6)), shows variations by a factor of 2 or less, peaking at the position DR21 C and around $(\mathrm{OH})$, i.e., towards the densest condensations along the ridge. This is remarkable since the $\mathrm{H}_{2}$ column density derived from the dust was found to vary by a factor of 12 . The column density for DR21 $(\mathrm{OH})$ of $6.3 \times 10^{17} \mathrm{~cm}^{-2}$, as determined by Zmuidzinas et al., is only slightly higher than our result.

\subsubsection{Carbon monoxide}

The column density of ${ }^{13} \mathrm{CO}$ is determined in two ways again assuming optically thin emission. First, by adopting $T_{\text {ex }}=79.4 \mathrm{~K} / \ln \left(4 / R^{13} \mathrm{CO}\right.$,high $)\left(\right.$ Eq. (C.9)) with the ${ }^{13} \mathrm{CO} 6-5$ to 3-2 integrated intensity line ratio $R_{13} \mathrm{CO}$,high, and using Eq. (C.10). This ratio is a sensitive temperature measure from $\sim 20 \mathrm{~K}$ up to a few $100 \mathrm{~K}$. The excitation temperatures vary between 30 and $46 \mathrm{~K}$ and represent upper limits because of possible self-absorption due to a high optical thickness in the lower transition. The temperatures agree with values from Wilson \& Mauersberger (1990), who find $20-65 \mathrm{~K}$ from $\mathrm{NH}_{3}$ emission, and are always below the $\mathrm{CI}$ temperatures from above, indicating an origin from different gas. The ${ }^{13} \mathrm{CO}$ column density, $23-54 \times 10^{15} \mathrm{~cm}^{-3}$, varies by a factor of 2 as the $C$ I column density.

The same scatter is found if we estimate the column density, in an alternative approach, from ${ }^{13} \mathrm{CO} 1-0$ rather than ${ }^{13} \mathrm{CO} 6-5$ (Eq. (C.11)) and derive temperatures with Eq. (C.8). Those column densities tend to be higher, between $33-76 \times 10^{15} \mathrm{~cm}^{-3}$, indicating that a larger amount of the gas traced by ${ }^{13} \mathrm{CO}$ is cold.

Considering the optically thin emission of $\mathrm{C}^{18} \mathrm{O}$, we estimated the total molecular gas column density from Eq. (C.12) using both ${ }^{13} \mathrm{CO} T_{\mathrm{ex}}$ estimates as limits. We found high $\mathrm{C}^{18} \mathrm{O}$ column densities of about $1-2 \times 10^{16} \mathrm{~cm}^{-2}$ along the ridge and $2-4 \times 10^{15} \mathrm{~cm}^{-2}$ toward $\mathrm{E}$ and $\mathrm{W}$.

\subsubsection{Ionised carbon}

In order to get a rough estimate of the $\mathrm{C}^{+}$column density from the ISO data, we assumed $N(\mathrm{C}$ II $)=6.4 \times 10^{20} \times I([\mathrm{C} \mathrm{II}])\left[\mathrm{cm}^{-2}\right]$ $\left(\mathrm{erg} \mathrm{s}^{-1} \mathrm{~cm}^{-2} \mathrm{sr}^{-1}\right)^{-1}$ (Crawford et al. 1985), which is a good approximation for an excitation temperature of $>91 \mathrm{~K}$ and a density $>n_{\mathrm{cr}}=5 \times 10^{3} \mathrm{~cm}^{-3}$. The peak column density derived this way, $N(\mathrm{C}$ II $) \sim 5.2 \times 10^{17} \mathrm{~cm}^{-3}$, is observed towards DR21 C and $\mathrm{E}$, while only about $28 \%$ of the peak value is found towards DR21 $(\mathrm{OH})$.

\subsubsection{Abundances}

The comparison between the $\mathrm{C}^{18} \mathrm{O}$ and ${ }^{13} \mathrm{CO}$ column density (for the lower $T_{\text {ex }}$ limit in Table 6) shows that the abundance ratio $\left[{ }^{13} \mathrm{CO}\right]:\left[\mathrm{C}^{18} \mathrm{O}\right]=6.6-11.4$ is typically near the canonical local abundance ratio [490]:[65] = 7.5 (Langer \& Penzias 1990).

The atomic carbon abundances $[\mathrm{C} \mathrm{I}] /\left[\mathrm{H}_{2}\right]$ fall in the range of $0.1-1.5 \times 10^{-5}$. The high values are only observed at the $\mathrm{E}$ and $\mathrm{W}$ positions. In the cooler northern positions, the abundance is reduced by a factor up to 10 . This contrasts with the values of $1-2 \times 10^{-5}$ found in cold dark clouds by Keene et al. (1997). 
Table 7. The physical parameters for the observed ISO/LWS positions. The radii listed in Col. 2 give the inner and outer edge of each shell. Columns 3 to 7 lists the temperature, clump-averaged number density of $\mathrm{H}_{2}$, volume filling, column density of $\mathrm{H}_{2}$, and the gas mass. For these columns, the first entry is the mass-averaged value for the whole cloud, values in parentheses show averages within each shell. The last column lists the reduced $\chi^{2}$ fit result for 11 lines (6 KOSMA, 2 FCRAO and 3 ISO).

\begin{tabular}{lccccccc}
\hline \hline Position & $\begin{array}{c}\text { Radii } \\
{[\mathrm{pc}]}\end{array}$ & $\begin{array}{c}T_{\text {gas }} \\
{[\mathrm{K}]}\end{array}$ & $\begin{array}{c}n\left(\mathrm{H}_{2}\right) \\
{\left[10^{4} \mathrm{~cm}^{-3}\right]}\end{array}$ & $\begin{array}{c}\phi_{\mathrm{v}}{ }^{a} \\
\text { volume filling }\end{array}$ & $\begin{array}{c}N\left(\mathrm{H}_{2}\right)^{b} \\
{\left[10^{21} \mathrm{~cm}^{-2}\right]}\end{array}$ & $\begin{array}{c}\text { Mass }^{c} \\
{\left[M_{\odot}(D / 1.7 \mathrm{kpc})^{2}\right]}\end{array}$ & $\chi_{0}^{2}$ \\
\hline DR21 E & $0.04 / 0.2 / 0.3$ & $62(87 / 55)$ & $92(300 / 4.8)$ & $0.04(0.01 / 0.89)$ & $46(9 / 37)$ & $243(46 / 197)$ & 2.2 \\
DR21 C & $0.12 / 0.2 / 0.6$ & $36(148 / 32)$ & $110(40 / 10)$ & $0.024(0.08 / 0.02)$ & $65(2 / 63)$ & $1379(51 / 1328)$ & 3.5 \\
DR21 W & $0.04 / 0.1 / 0.3$ & $54(118 / 52)$ & $12(100 / 9)$ & $0.59(0.06 / 0.8)$ & $88(3 / 85)$ & $489(15 / 474)$ & 2.8 \\
DR21 (OH) & $0.04 / 0.2 / 0.7$ & $31(99 / 29)$ & $161(220 / 160)$ & $0.01(0.01 / 0.01)$ & $49(1 / 48)$ & $1421(34 / 1387)$ & 3.9 \\
DR21 FIR1 & $0.04 / 0.2 / 0.5$ & $41(82 / 37)$ & $16(220 / 2)$ & $0.1(0.01 / 1)$ & $47(4 / 43)$ & $700(59 / 641)$ & 4.1 \\
\hline
\end{tabular}

${ }^{a}$ The product of the volume filling and the abundance of the species is one of the free parameters. We fixed the abundance $\left[X / \mathrm{H}_{2}\right]$ of ${ }^{12} \mathrm{CO}$ to $2 \times 10^{-4}$ (resp. $3.1 \times 10^{-6}$ for ${ }^{13} \mathrm{CO}$ and $4 \times 10^{-7}$ for $\mathrm{C}^{18} \mathrm{O}$ ). ${ }^{b}$ Column densities are source-averaged and not normalized to $80^{\prime \prime} .{ }^{c}$ The molecular gas mass is derived from the cloud-averaged density. This density is the product of local $\mathrm{H}_{2}$ density of the clumps and volume filling correction $\phi_{\mathrm{v}}$.

Except for DR21 FIR1, there is a good correlation between $X(\mathrm{CI})$ and $X\left(\mathrm{C}^{18} \mathrm{O}\right)$. Toward DR2 $1(\mathrm{OH})$, the $\mathrm{C}^{18} \mathrm{O}$ and [C I] abundances are systematically lower than toward DR21 by factors $2-3$, although the position with the lowest [CI] abundance $\left(10^{-6}\right)$, FIR1, has the highest $\mathrm{C}^{18} \mathrm{O}$ abundance (up to $\left.3.7 \times 10^{-7}\right)$.

Mookerjea et al. (2006) present a compilation of the $\mathrm{C} / \mathrm{CO}$ abundance ratios versus $\mathrm{H}_{2}$ column densities for Galactic starforming regions and diffuse clouds. The ratio at the positions DR21 E, W, and FIR1, 0.07-0.17, agrees well with the relation $\log [N(\mathrm{C}) / N(\mathrm{CO})]=-0.94 N\left(\mathrm{H}_{2}\right)+19.9$ given there for Cepheus B. DR21 and DR21 $(\mathrm{OH})$ deviate from this relation but are still within the observed scatter. We therefore underline their conclusion that $\mathrm{C} \mathrm{I}$ is no straightforward tracer of total $\mathrm{H}_{2}$ column densities and total masses.

The amount of carbon in the carbon bearing species $\mathrm{C}^{+}, \mathrm{C}^{0}$, and $\mathrm{CO}$ can be compared - by using their relative abundances (last row of Table 6). The neutral atomic carbon relative abundance is rather homogeneous at about $9-16 \%$ of the total carbon gas content at all five positions. The rest is shared between $\mathrm{CO}$ and $\mathrm{C}$ II. Ionised carbon has the highest abundance toward DR21 E (26\%). Here, the gas is very likely exposed to a high UV radiation field and carbon species are photodissociated to $\mathrm{C}$ I and ionised further to $\mathrm{C}$ II. High fractional $\mathrm{CO}$ abundances $(>85 \%)$, such as for DR21 (OH) and FIR1, were also reported for S 106 ( $>86 \%$, Schneider et al. 2003) and W3 Main (>60\%, Kramer et al. 2004). The ratios in other sources are, e.g., 37:07:56 for IC 63 (Jansen et al. 1996), NGC2024 with 40:10:50 (Jaffe \& Plume 1995), or $\eta$ Carina with 68:15:16 (Brooks et al. 2003).

\subsection{Radiative transfer modeling}

In a self-consistent approach, the temperature, column and number density, total molecular mass, volume filling, and mass composition were determined using a 1D radiative transfer code (SIMLINE, Ossenkopf et al. 2001). In order to describe these physical conditions, we created heterogeneous models with internal clumping and turbulence in a spherically symmetric geometry for each of the 5 observed regions. The physical parameters of a shell are defined by their values at the inner radius, and the radial dependence is described by a power-law.

A model with two nested shells provided reasonable results in most cases: (i) a hot and dense molecular interface zone wrapped around a central H II-region; (ii) a massive cold cloud, the envelope region, enclosing the interface region. While in the inner component primarily the mid- and high- $J$ CO emission lines are excited, this envelope is responsible for self-absorption and the low lying lines of ${ }^{13} \mathrm{CO}$ and $\mathrm{C}^{18} \mathrm{O}$. We set the abundance of species with respect to molecular hydrogen to standard values (see Table 7). The source size is a crucial parameter that is not well-confined by the line profiles or the map we obtained. A small cloud model can easily produce similar results to those for a bigger cloud with a lower volume filling. We therefore approximated the radii using high-resolution line and continuum observations (Wilson \& Mauersberger 1990; Lane et al. 1990; Jaffe et al. 1989; Chandler et al. 1993; Vallée \& Fiege 2006). The inner cut-off was assumed to be $0.04 \mathrm{pc}\left(<10^{4} \mathrm{AU}\right)$, except for DR21 C where we estimated $\sim 0.12$ pc from the $14.7 \mathrm{GHz}$ continuum (Roelfsema et al. 1989). The H II-region is considered to be free of molecular gas, so we neglected the continuum contribution by ionised gas. Gradients within the shells in any of the parameters were initially set to zero and were adjusted during the fit if required.

We used the following recipe to find appropriate model parameters. (i) The linewidth is approximated by adjusting the turbulent velocity field until both the low- $J$ and mid- $J$ line wings match the observed profile. As line emission from the inner warm gas is shielded by the colder envelope gas closer to the surface, the width of the $\mathrm{CO}$ absorption dip in the line centre constrains the turbulent velocity dispersion of this envelope component; (ii) the $\mathrm{H}_{2}$-density is defined locally as a clumpdensity parameter, and was varied independently between $10^{4}$ and $10^{7} \mathrm{~cm}^{-3}$ for both shells. The high densities are a prerequisite for high- $J \mathrm{CO}$ excitation; (iii) in the optically thin line wings we see material from the inner shell predominantly where the simulated macroturbulent motion leads to the higher line width which is only partly shielded by the outer shell. This allows us to fit the temperatures of both the inner and the outer shells from the line shape; (iv) the model allows us to differentiate between radial and turbulent gas motion. Where lines of high opacity toward the line centre showed a blue-skewed profile, we introduced radial infall as a plausible explanation. If possible, we tried to keep the radial component close to zero.

The full line profiles of $\mathrm{CO},{ }^{13} \mathrm{CO}$, and $\mathrm{C}^{18} \mathrm{O}$ from KOSMA and FCRAO were taken into account in a reduced $\chi^{2}$ fit in $\sim 1900$ degrees of freedom with 20-28 free parameters. The second velocity component is masked out by a window centred on $9 \pm 3 \mathrm{~km} \mathrm{~s}^{-1}$. For the ISO lines, we fit the integrated flux of three lines. CO 14-13 is not considered because no consistent fit was possible in conjunction with the other CO lines. In total, we included 11 lines weighted by the $\sigma^{2}$ determined from a baseline region not including any lines. The spectra do not contain any obvious baseline ripples above the system noise. 


\subsubsection{Line modeling: $\mathrm{CO},{ }^{13} \mathrm{CO}$, and $\mathrm{C}^{18} \mathrm{O}$}

We independently optimized the model parameters for each position. Figure 5 shows the derived line profiles. At all five positions, we obtained solutions of better than $\chi_{0}^{2}=4.1$, which we consider - given the number of fitted lines - a very good result and definitely are much better than for a single-component model.

Within the interface-zone, a temperature range from $80 \mathrm{~K}$ (at DR21 FIR1) to $148 \mathrm{~K}$ (at DR21 C) for gas masses of $15-60 M_{\odot}$ is found. This temperature is much higher than the typical LTE excitation temperature of the mid- $J$ CO line peak. The fit converges toward solutions with a high density of about $10^{6} \mathrm{~cm}^{-3}$ and with a volume filling of a few percent, indicating a nonhomogeneous distribution. The warm gas dominates the emission in the line wings and therefore is explained by the steep turbulent velocity gradient with velocities up to $30 \mathrm{~km} \mathrm{~s}^{-1}$. Because the envelope is much colder and more massive (cf. Sect. 4.2.3), the average temperature in the regions DR2 $1 \mathrm{C}$ with $36 \mathrm{~K}$ and $\mathrm{DR} 21(\mathrm{OH})$ with $31 \mathrm{~K}$ is rather low compared to the $\mathrm{E}, \mathrm{W}$, and FIR 1 position. There, the gas temperature rises up to $60 \mathrm{~K}$, but the gas masses are only a few hundred solar masses. Besides the mass fraction, the steepness of the gradient in excitation condition and line width between inner and outer components determines whether we see a distinct self-absorption (e.g., DR21 C) or a flat-top (e.g., DR21 W) line profile. Table 7 compiles a list of the derived physical parameters. A detailed discussion of the individual positions is given in Appendix A.

\subsubsection{Line modeling: $[\mathrm{Cl}]$ and $\mathrm{CS}$}

As a consistency check, we computed the CS and C I profiles using the same model parameters as derived from CO. This implies a homogeneous distribution of CS and C I with CO, which is only affected by the abundances of the species.

For both species we also obtained excellent fit results. The resulting line profiles are shown in Fig. 5. CS 7-6 is only excited where the $\mathrm{H}_{2}$ density and temperatures are sufficiently high; hence, we only find CS emission in the dense regions DR21 C and $(\mathrm{OH})$. The models fit best to the observations for an abundance of $X(\mathrm{CS})=4 \times 10^{-9}$ (Hatchell et al. 1998), whereas the range $1 \times 10^{-10}$ to $2.3 \times 10^{-10}$ given by Shirley et al. (2003) is clearly too low for this region.

Instead of a carbon abundance variation across the positions (as found in Sect. 4.1), an abundance $X(\mathrm{CI})$ of $1 \times 10^{-5}$ fits our $[\mathrm{CI}$ ] observations nicely. The $\mathrm{CI}$ excitation temperatures, as derived in Sect. 4.1.1, fall in the range covered by the twocomponent radiative transfer model.

\subsubsection{Masses and volume filling}

The volume filling, $\phi_{\mathrm{v}}$, is a model parameter capable of increasing the clump density without increasing the overall mass as well. The determined $\phi_{\mathrm{v}}$ is usually low between $1 \%$ to $8 \%$ (Col. 5 in Table 7). At DR21 C, this is confirmed by our observations of the CS 7-6 transition, which is sensitive to densities of several $10^{6} \mathrm{~cm}^{-3}$ : Given the high $\mathrm{H}_{2}$ column density (up to $2 \times 10^{23} \mathrm{~cm}^{-2}$ ), this translates to a column-to-volume density ratio of about $0.006 \mathrm{pc}$. If we assume that the visible extent of the DR21 source $(\sim 0.69 \mathrm{pc}$ in CS, after deconvolving the beam) is similar to the size along the line-of-sight, a volume filling of $\sim 0.01$ is derived. A high degree of small-scale spatial and velocity structure is also needed to explain the hyper-finestructure anomalies observed in ammonia data toward DR21
(Stutzki 1985). Shirley et al. (2003), who studied CS 5-4 in a sample of high-mass star-forming regions, derived a volume filling with a median of 0.14 . This is one order of magnitude higher than our results for the dense regions DR21 and DR21 $(\mathrm{OH})$.

The total mass for all 5 positions is $4.2 \times 10^{3} M_{\odot}$, which is mostly shared between DR21 C and DR21 (OH) with $\sim 1.4 \times$ $10^{3} M_{\odot}$ each. In direct comparison to the masses derived from dust observation in Sect. 3.3.1, we find that our model gives higher masses $(\times 1.6-\times 1.8)$, except for the DR21 $(\mathrm{OH})$ model mass. To compare our mass estimate with masss from the literature, often derived for a different distance, we scaled the mass in Table 7 with the square of the distance, basically assuming that the column density, computed from the observed lines, is independent of the distance. This approach is consistent with a local radiative transfer treatment, such as LTE or escape probability analyses, where the molecular excitation does not depend on the total gas mass. For the self-consistent radiative transfer code, this assumption is, however, not completely justified, but deviations from this simple scaling relation are to be expected. When comparing the values from Table 7 to results obtained for much larger distances, our quadratically scaled mass estimates are therefore always expected to be too high; and when comparing them to results for lower distances, they should be systematically lower. Shirley et al. estimated virial masses of $561 M_{\odot}(D / 1.7 \mathrm{kpc})$ for DR21 (resp. $714 M_{\odot}(D / 1.7 \mathrm{kpc})$ for DR21 $(\mathrm{OH})$ ). These estimates are lower than our masses but were calculated over a much smaller radius of $0.17 \mathrm{pc}(D / 1.7 \mathrm{kpc})$, while our region covers up to $0.7 \mathrm{pc}$. Ossenkopf et al. (2001) estimated the DR21 mass using a radiative transfer analysis of CS data. They estimated $578 M_{\odot}(D / 1.7 \mathrm{kpc})^{2}$ at a (cloud-averaged) density of $1.6 \times 10^{3} \mathrm{~cm}^{-3}$. Their lower density is partly explained by the larger outer radius, which was chosen to be $1.0 \mathrm{pc}(D / 1.7 \mathrm{kpc})$. It is also probably due to the larger physical cloud size at the assumed distance of $D=3 \mathrm{kpc}$, which prevents the simple quadratic distance scaling of the mass in a self-consistent radiative-transfer treatment.

The total mass of DR2 $1(\mathrm{OH})$ corresponds to a cloudaveraged column density of $\sim 5 \times 10^{22} \mathrm{~cm}^{-2}$. The value of $5.7 \times$ $10^{23} \mathrm{~cm}^{-2}$ reported by Richardson et al. from $1100 \mu \mathrm{m}$ data and our value given in Table 5 based on the far-IR dust-emission are higher by a factor of 5-10. A flatter spectral index $\beta$ may explain why dust and line-observation analyses result in different conclusions. Values between 1.0 (Vallée \& Fiege 2006) and 2.0 (Chandler et al. 1993) were discussed for DR21 (OH) in the literature. If $\beta$ is lower than the assumed 1.5, this will have a significant effect on the derived $A_{\mathrm{v}}$. For instance, with $\beta=1.25$, the column density is reduced to about one fourth of our value at $\beta=1.5$, so is then consistent with the CO-model results.

An alternative explanation of the discrepancy could be depletion as a result of a freeze-out of $\mathrm{CO}$ onto grains. Some authors (e.g., Vallée \& Fiege 2006, and references therein) use a depletion factor of 10 or more $\left(X(\mathrm{CO})=2.5 \times 10^{-6}\right.$ at DR2 $\left.(\mathrm{OH})\right)$ as appropriate for star-forming clouds. In Jørgensen et al. (2004), the abundance is reduced by a factor of 10 for the conditions $T<30 \mathrm{~K}$ and $n>3 \times 10^{4} \mathrm{~cm}^{-2}$. The DR21 (OH) envelope and the resulting column density would comply with these conditions. However, the $\mathrm{CO}$ line data analysis does not allow us to draw conclusions toward depletion.

\subsubsection{Problems of the fit}

As noted before, a precise determination of the outer radii of the model clouds, hence their mass, is quite uncertain, and a small increase may already more than double the mass. In the 


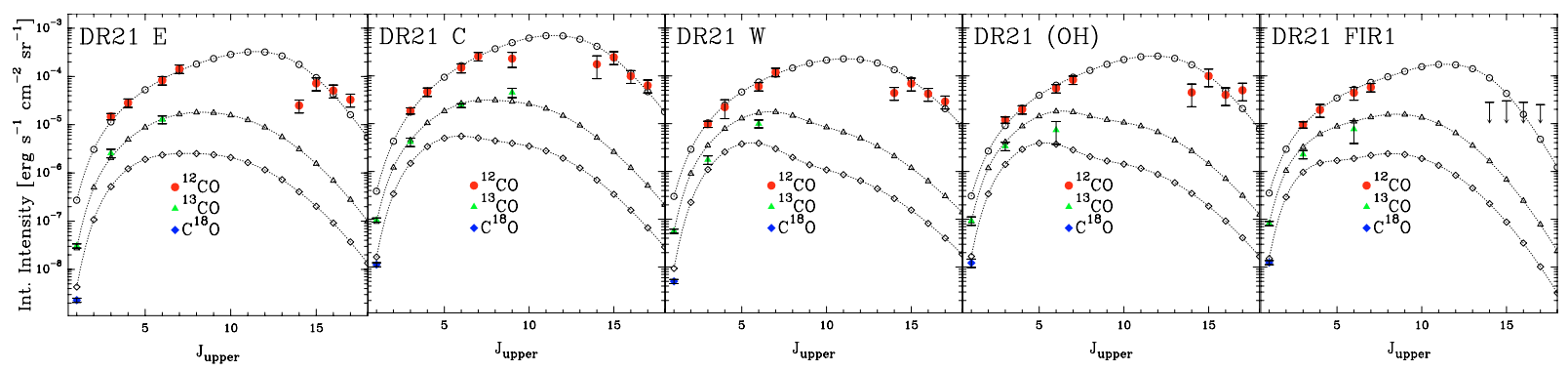

Fig. 8. Cooling curves of the best-fit model line fluxes (marked by open symbols) of $\mathrm{CO},{ }^{13} \mathrm{CO}$, and $\mathrm{C}^{18} \mathrm{O}$. Observed line intensities are indicated as filled symbols (see Table 3). At position DR21 C, we included two observations by Boreiko \& Betz (1991) of CO and ${ }^{13} \mathrm{CO} J=9-8$. Since the ${ }^{13} \mathrm{CO} 6-5$ map does not cover the postitions DR21 (OH) and DR21 FIR1 on a fully sampled grid, we expect a larger error. The high- $J$ CO emission at DR21 FIR1 are upper limits.

models of DR21 C and DR21 $(\mathrm{OH})$, we found no reasonable solutions for radii much smaller than $0.6 \mathrm{pc}$ matching the ${ }^{13} \mathrm{CO}$ and $\mathrm{C}^{18} \mathrm{O} 1-0$ observations. As a possible indication of a systematic error in the column density, we typically see that the observed peak temperature of the $\mathrm{C}^{18} \mathrm{O} 1-0$ line is weaker than predicted by the models (see Fig. 5) while the ${ }^{13} \mathrm{CO} 1-0$ line is reproduced well. This can either be a result of an inaccurate modeling of density and temperature conditions, or our assumption about the fixed abundances for $\mathrm{CO},{ }^{13} \mathrm{CO}$, or $\mathrm{C}^{18} \mathrm{O}$ is incorrect.

The models overestimate the ${ }^{13} \mathrm{CO} 3-2$ peak temperature. Except for DR21 E, this transition is optically thick $(\tau>2)$ and reflects the kinetic temperature of the envelope gas. As the CO 3-2 to 7-6 lines also become optically thick in the envelope, the brightness temperature at the self-absorbing line centre of these mid- $J$ lines is governed by the same material. The very deep $(\sim 5 \mathrm{~K})$ absorption dip in CO 7-6 observed by Jaffe et al. (1989) indicates very cold foreground gas $(<20 \mathrm{~K})$, which we cannot reproduce with only one component for the outer shell. A better match of the observed intensities may be possible by adding a third colder layer outside of the envelope.

Since the cold and warm components certainly are intermixed to some extent rather than separated into two nested shells, the focus during the modeling was put on a qualitative description of the different components and should not be seen as a geometric representation of the real cloud structure. We demonstrated, however, that the simplified assumption of a spherical cloud is adequate for estimating physical properties, such as mass, density, filling factors, and temperature.

\subsection{Oxygen and nitrogen}

\subsubsection{ISO/LWS far-IR lines: [O I], [O III], and [N II]}

In addition to the high- $J \mathrm{CO}$ lines (see Sect. 3.3.2), ISO provided the integrated line intensities of [O I] (63 and $145 \mu \mathrm{m}$ ) (Table 4). Their relative strength and cooling efficiency depends on the temperature and density regime: At low temperatures, rotational $\mathrm{mm}$ - and submm-lines of $\mathrm{CO}$ dominate the cooling of the molecular and neutral gas. At temperatures above $T \sim 150 \mathrm{~K}$, pure rotational transitions of $\mathrm{OH}, \mathrm{H}_{2} \mathrm{O}, \mathrm{H}_{2}$, and the $63 \mu \mathrm{m}$ [O I] line become more important. The [O I] at $63 \mu \mathrm{m}$ and the atomic and ionic fine-structure lines of [C II] and [C I] are diagnostic lines for PDRs. Due to the nearby H II-regions, the ionic lines [O III] and $[\mathrm{N} \mathrm{II}]$ are also expected to be strong emitters and may serve as diagnostics to distinguish the fraction of [C II] emission coming from the neutral and ionised gas. For completeness, we included the [O III] (52 and $88 \mu \mathrm{m})$ and [N II] $(122 \mu \mathrm{m})$ lines in the table.
Since DR21 E, C and $\mathrm{W}$ are lined up along the outflow axis, we compared the emission in the outflow lobes with the central position. The strong [OI] emission at $63 \mu \mathrm{m}$ decreases rapidly toward the west (cf., Lane et al. 1990). This line easily becomes optically thick and Poglitsch et al. (1996) indeed found a deeply absorbed line toward DR21. The $63 \mu \mathrm{m}$ emission extends toward the E position at almost the same strength as toward the centre. As the $A_{\mathrm{v}}$ at the same time drops down, the gas traced by OI must be a separate, necessarily warm component. The [OI] line at $145 \mu \mathrm{m}$ is peaked at DR21 C, showing only a slight east-west asymmetry.

If we assume thermalized optically thin [OI] emission, we can derive the excitation temperature from $T_{\mathrm{ex}}=$ $227.7 \mathrm{~K} / \ln \left(2.83 /\left(12.22 \cdot R_{[\mathrm{O} I]}\right)\right)\left(\mathrm{Eq}\right.$. (C.13)), where $R_{[\mathrm{O} \text { I }]}$ is the ratio of [O I] 145/63 $\mu \mathrm{m}$ emission (cf. Table 4). The ratios of observed intensities, $0.06-0.12$, result in temperatures between 170 to $370 \mathrm{~K}$ and are to be seen as strict upper limits, since $R_{[\mathrm{O} \text { I] }}$ decreases for optically thick [OI] $63 \mu \mathrm{m}$ line emission. The [O I] line ratio at $\mathrm{DR} 21(\mathrm{OH})$ of $R_{[\mathrm{O} \mathrm{I}]}=0.29$ requires a temperature just below $100 \mathrm{~K}$. We detect the weakest emission of [O I] at DR21 FIR1. Errors in $R_{[\mathrm{O} I]}$ become too large to estimate a temperature here.

As expected for the position of the HII-region, both ionised [O III] lines are strong at DR21 C and decrease toward the sides. Furthermore, the [O III] $88 / 52 \mu \mathrm{m}$ line ratio is a good tracer of the electron density in the range $10^{2}-10^{5} \mathrm{~cm}^{-3}$, while being insensitive to abundance and temperature variations (Rubin et al. 1994). At DR21 C, a ratio of about 0.4 indicates an electron density of $\left\langle N_{\mathrm{e}}\right\rangle \sim 10^{3} \mathrm{~cm}^{-3}$. In the east, the ratio rises to 0.8 indicating an extension of the H II-region to this side. To the west, the ratio is above 1.4. An electron density below $10^{2} \mathrm{~cm}^{-3}$, consistent with the observed [O III] line ratio at DR21 (OH), indicates a low degree of ionisation. No H II-region has been identified in this area, e.g., in the VLA 6-cm continuum (Palmer et al. 2004). Thus, the finding of $[\mathrm{OIII}]$ emission is a bit surprising. The [O III] ratio $>3 \pm 1.3$ found at DR21 FIR1 is not covered by the Rubin et al. model but corresponds to a very low $\left\langle N_{\mathrm{e}}\right\rangle$.

The [N II] $122 \mu \mathrm{m}$ line was only detected at the $\mathrm{E}$ and $\mathrm{W}$ positions, while the $3 \sigma$ noise limit at the position of the H II-region DR21 C still permits centrally-peaked emission. The fraction of [C II] stemming from the H II-region can be estimated from the intensity ratio of [C II] to [N II]. The Galactic abundance ratio $\mathrm{C} / \mathrm{N}$ in dense $\mathrm{H}$ II-regions leads to an expected ratio of $[\mathrm{C} \mathrm{II}] /[\mathrm{N} \mathrm{II}]=1.1$ (Kramer et al. 2005, and references therein). With the observed [C II]/[N II] ratio between 11-19 for DR21, less than $10 \%$ of the $[\mathrm{C} \mathrm{II}]$ emission is estimated to come from the ionised gas. 
Table 8. Total cooling intensities of $\mathrm{CO},[\mathrm{CI}],\left[\mathrm{CII}\right.$, and [O I] . The values in parentheses show the relative contribution to the sum $I_{\text {tot }}$. The total cooling efficiency $\epsilon_{\mathrm{tot}}$ is defined by $I_{\mathrm{tot}} / I_{\mathrm{IR}}$. For comparison, we also list the $\epsilon^{\prime}$ defined by $\left(I_{[\mathrm{CII}}+I_{[\mathrm{OI}]}\right) / I_{\mathrm{IR}}$.

\begin{tabular}{|c|c|c|c|c|c|}
\hline Intensity in $\left[10^{-4} \mathrm{erg} \mathrm{s}^{-1} \mathrm{~cm}^{-2} \mathrm{sr}^{-1}\right]$ & $\overline{\text { DR21E }}$ & $\overline{\mathrm{DR} 21 \mathrm{C}}$ & $\overline{\mathrm{DR} 21 \mathrm{~W}}$ & $\overline{\mathrm{DR} 21(\mathrm{OH})}$ & $\overline{\text { DR21 FIR1 }}$ \\
\hline$I_{\mathrm{CO}}\left(=\Sigma \mathrm{CO}_{1 \leq J_{\mathrm{u}} \leq 20}\right)^{a}$ & $24.1(28.8 \%)$ & $51.3(44.4 \%)$ & $18.7(53.7 \%)$ & $20.0(75.9 \%)$ & $13.4(68.7 \%)$ \\
\hline$I_{\left[\mathrm{C}_{\mathrm{I}}\right]}(=\Sigma[\mathrm{C} \mathrm{I}])^{b}$ & $0.22(0.3 \%)$ & $0.45(0.4 \%)$ & $0.23(0.7 \%)$ & $0.25(0.9 \%)$ & $0.27(1.4 \%)$ \\
\hline$I_{[\mathrm{O} I]}(=\Sigma[\mathrm{OI}])^{b}$ & $51.1(61.1 \%)$ & $55.6(48.2 \%)$ & $11.2(32.1 \%)$ & $3.78(14.4 \%)$ & $2.23(11.4 \%)$ \\
\hline$I_{[\mathrm{C} \mathrm{II}]}$ & $8.2(9.8 \%)$ & $8.1(7.0 \%)$ & $4.7(13.5 \%)$ & $2.3(8.8 \%)$ & $3.6(18.5 \%)$ \\
\hline$I_{\mathrm{tot}}$ & $83.62(100 \%)$ & $115.45(100 \%)$ & $34.83(100 \%)$ & $26.33(100 \%)$ & $<19.5(100 \%)$ \\
\hline$I_{\mathrm{IR}}^{c}$ & 5500 & 33900 & 1810 & 12700 & 3680 \\
\hline$\epsilon_{\mathrm{tot}}[\%]$ & 1.5 & 0.34 & 1.9 & 0.21 & 0.52 \\
\hline$I_{\left[\mathrm{C}_{\mathrm{II}}\right]+[\mathrm{OI}]}\left([\mathrm{C} \mathrm{III}]+[\mathrm{O} \mathrm{I}]_{63} \mu \mathrm{m}\right)$ & 56.4 & 57.5 & 14.8 & 5.24 & 5.38 \\
\hline$\epsilon^{\prime}[\%]$ & 1.0 & 0.17 & 0.82 & 0.04 & 0.15 \\
\hline
\end{tabular}

${ }^{a}$ For CO we use the best fitting model (Sect. 4.2) and sum over all rotational lines up to $J=20 .{ }^{b}$ For C I and O I, we sum the observed intensities of both fine structure lines. ${ }^{c} I_{\mathrm{IR}}$ is the total observed infrared intensity (Table 5).

\subsubsection{Line modeling: [O I] and [C II]}

A determination of the $\mathrm{O}$ I and $\mathrm{C}$ II abundances may be difficult. If we assume that their abundance is closely connected to the dense molecular gas, we can calculate and fit the emission of these two species by adjusting the abundance of the two-shell radiative transfer models (Sect. 4.2). At DR21 C, we find abundances of $6 \times 10^{-5}$ for O I and $2.5 \times 10^{-4}$ for C II. For both C II and the OI $63 \mu \mathrm{m}$ line, the opacity toward the line centre is about 9 , and the line profile is deeply self-absorbed. The high energy above the ground level of $\mathrm{O}$ I leads to almost complete absorption of the centre emission leaving signatures of warm gas only in the wings. The $145 \mu \mathrm{m}$ transition $(\tau \sim 0.5)$ is unaffected and is a better measure of the abundance. As C II has only one transition and also shows some absorption in the model profiles, the abundance may be less accurate. At DR21(OH), the abundance of $\mathrm{O}$ I is $\sim 1.2 \times 10^{-5}$ and $9 \times 10^{-5}$ for $\mathrm{C}$ II. The abundances at all five observed positions vary between $1-6 \times 10^{-5}$ for O I and $1-4 \times 10^{-4}$ for C II.

Compared to our LTE estimate in Sect. 4.1, the C II amount derived here is higher by factors of 13-160. Because of recombination reactions, it is rather unlikely to have a large amount of $\mathrm{C}$ II hidden in the cold gas, so this can be considered as an upper limit.

\section{Line cooling efficiency}

The relative importance of the various line tracers among each other and with respect to the FIR continuum provides a detailed signature of the heating and cooling processes in the DR21 molecular cloud. Table 8 lists the cooling intensities at the five selected ISO positions E, C, W, OH, and FIR1.

The total line cooling intensity, $I_{\text {tot }}$, derived by summing the contributions of CO, C I, C II, and O I, peaks at position C, dropping to less than $15 \%$ of this peak value at FIR1.

The relative contributions to $I_{\text {tot }}$ vary strongly at the 5 positions. [O I] is the strongest coolant at positions $\mathrm{E}$ and $\mathrm{C}$, while $\mathrm{CO}$ is strongest at $\mathrm{W},(\mathrm{OH})$, and FIR1, tying up more than $50 \%$ of the total cooling. The relative contribution of $\mathrm{CO}$ to $I_{\text {tot }}$ varies between $26 \%$ at $\mathrm{E}$ and $76 \%$ at $(\mathrm{OH})$, while the relative contribution of $[\mathrm{C} \mathrm{II}]$ is less, varying between $7 \%$ at $\mathrm{C}$ and $21 \%$ at FIR1. The two [CI] fine-structure lines contribute generally less than $2 \%$. The ratio of $\mathrm{CO}$ cooling relative to $[\mathrm{CI}]$ cooling peaks at $\mathrm{C}$ at 114 and drops to less than 50 at FIR1.

We also compared $I_{\text {tot }}$ with the FIR continuum to derive the gas cooling efficiency $\epsilon_{\mathrm{tot}}$ as a measure of the fraction of FUV photon energy which goes into gas heating. The value of $\epsilon_{\mathrm{tot}}$ varies by one order of magnitude, between $0.2 \%$ at $(\mathrm{OH})$ and $1.5-2 \%$ at the two outflow positions $\mathrm{E}$ and $\mathrm{W}$.

Table 8 also lists the cooling efficiency $\epsilon^{\prime}$ only taking the $158 \mu \mathrm{m}[\mathrm{CII}]$ and the $63 \mu \mathrm{m}$ [O I] lines into account, ignoring $\mathrm{CO}$ and other species, as is often done in the literature. Then, $\epsilon^{\prime}$ varies between $0.04 \%$ at $(\mathrm{OH})$ and $1 \%$ at $\mathrm{E}$.

Several studies in the literature have determined the gas heating efficiency $\epsilon^{\prime}$ in Galactic star-forming regions and in external galaxies. Vastel et al. (2001) find values typically of $0.2-0.4 \%$ in Galactic star-forming regions, $1.1 \%$ at the Orion Bar, and less than $0.035 \%$ toward W49N. For W3 Main/IRS5, Kramer et al. (2004) report $\epsilon^{\prime}$ of $\sim 0.1 \%$ similar to the value found in DR21 C. Malhotra et al. (2001) find efficiencies between 0.05 and $0.3 \%$ in 60 unresolved normal galaxies. Kramer et al. (2005) find efficiencies between 0.21 and $0.36 \%$ in the centres and spiral-arm positions of M 83 and M 51.

The PDR models, e.g., by Kaufman et al. (1999), predict the heating efficiency $\epsilon^{\prime}$ of a cloud illuminated from all sides between $0.01 \%$ to $1.5 \%$, and our values are within these theoretical limits.

A remarkable point is that only a few percent of the mass (up to $20 \%$ at DR21 E) is efficiently heated. Considering only the cold envelope component of our radiative transfer models, i.e., removing the warm interface zone, shows that the heated gas accounts for most of the CO emission (80\% to 94\%, summing over $J_{\text {up }}=1 \ldots 20$ ) in terms of radiated energy.

Since the total line power of all lines that we consider, $I_{\text {tot }}$, does not include the contributions from other cooling lines (e.g., of $\mathrm{H}_{2}, \mathrm{H}_{2} \mathrm{O}, \mathrm{OH}$, and PAHs), we may miss some of the cooling energy.

\section{Summary}

We have presented the first comprehensive mapping of sub-mm lines in the whole DR21/DR21 $(\mathrm{OH})$ region. The line emission of [C I] at $492 \mathrm{GHz}$ and $809 \mathrm{GHz}, \mathrm{CO} 3-2,4-3,6-5$, and 7-6, ${ }^{13} \mathrm{CO} 3-2$ and $6-5$, and of CS $7-6$ reveals contributions from different gas components: (i) a very wide-winged emission in mid- $J$ CO transitions having a line width of more than $15 \mathrm{~km} \mathrm{~s}^{-1}$ and self-absorption; and (ii) narrow (4 to $6 \mathrm{~km} \mathrm{~s}^{-1}$ ) emission in the other lines. Based on these lines, and supported by mm- and FIR-lines from FCRAO and ISO, we confined temperature, density, masses, and volume filling for five positions toward DR21, DR21 $(\mathrm{OH})$, and DR21 FIR1 using a radiative transfer code by modeling the spectral line profiles. 
1. We found that 2-component models consisting of a warm inner region and a cold envelope are needed to explain the observed strengths of the energetically low and high lying transitions of $\mathrm{CO}$ and to explain the self-absorption.

2. The bulk of molecular gas at DR21 and DR21 (OH) is cold $(\sim 30 \mathrm{~K})$ and concentrated in the ridge. The clump volume filling is typically a few percent and the density within a clump is of the order $10^{6} \mathrm{~cm}^{-3}$.

3. By fitting the dust emission SED, we constrained the $\mathrm{H}_{2}$ column density to $2-5 \times 10^{22} \mathrm{~cm}^{-3}$ for DR21 E, W and toward FIR1. At DR21/DR21 (OH) it rises to above $10^{23} \mathrm{~cm}^{-3}$. The dust temperatures vary between $31-43 \mathrm{~K}$ and are close to the temperature of the cold gas.

4. Warm gas (80-150 K) was found at all five positions. The warm gas mass is typically a few percent of the total mass, but it accounts for most of the total $\mathrm{CO}$ emission $(80 \%$ to $94 \%$, mainly in the mid- and high- $J$ tranitions). As the coldest source, DR21 $(\mathrm{OH})$ still shows a significant amount of high- $J \mathrm{CO}$ emission.

5. CS 7-6 emission can be reproduced if the local density in the envelope region is sufficiently high. Therefore, high densities are expected at DR21 and DR21 (OH), again supporting the low volume-filling factors.

6. Supported by the model results, we derived the total intensity of the strongest emission lines ([O I $], \mathrm{CO},[\mathrm{C} \mathrm{II}]$, and [C I]). While the gas is predominantly cooled by emission of dust grain, these lines provide up to to $2 \%$ of the total cooling of the atomic and molecular gas. At the dense cores DR21 and DR21 $(\mathrm{OH})$, the cooling efficiency is less than $0.5 \%$. At DR21, O I and CO are equally strong, whereas at DR21 (OH) the $\mathrm{CO}$ lines dominate the cooling by more than $75 \%$. C II contributes generally less than $20 \%$, and C I not more than $1.5 \%$, to the line cooling. The [O I] emission shows a large variation from $<15 \%$ at DR2 $(\mathrm{OH})$ and FIR 1 to $61 \%$ at $\mathrm{E}$.

Acknowledgements. The KOSMA $3 \mathrm{~m}$ submillimeter telescope at the Gornergrat-Süd is operated by the University of Cologne in collaboration with the Radio Astronomy Department of the Argelander-Institute for Astronomy (Bonn) and supported by special funding from the Land NRW. The observatory is administrated by the International Foundation Gornergrat \& Jungfraujoch.

\section{References}

Black, J. H. 2000, in Astrochemistry: From Molecular Clouds to Planetary, ed. Y. C. Minh, \& E. F. van Dishoeck, 81

Bohlin, R. C., Savage, B. D., \& Drake, J. F. 1978, ApJ, 224, 132

Boreiko, R. T., \& Betz, A. L. 1991, ApJ, 369, 382

Bradford, C. M., Nikola, T., Stacey, G. J., et al. 2003, ApJ, 586, 891

Bradford, C. M., Stacey, G. J., Nikola, T., et al. 2005, ApJ, 623, 866

Brooks, K. J., Cox, P., Schneider, N., et al. 2003, A\&A, 412, 751

Calzetti, D., Armus, L., Bohlin, R. C., et al. 2000, ApJ, 533, 682

Campbell, M. F., Niles, D., Nawfel, R., et al. 1982, ApJ, 261, 550

Cernicharo, J. 1985, ATM: A program to compute theoretical atmospheric opacity for frequencies $<1000 \mathrm{GHz}$, Tech. rep., IRAM

Chandler, C. J., Gear, W. K., \& Chini, R. 1993, MNRAS, 260, 337

Clegg, P., Ade, P., Armand, C., \& Baluteau, J.-P. 1996, A\&A, 315, 38

Crawford, M. K., Genzel, R., Townes, C. H., \& Watson, D. M. 1985, ApJ, 291, 755

Cyganowski, C. J., Reid, M. J., Fish, V. L., \& Ho, P. T. P. 2003, ApJ, 596, 344

Davis, C. J., Kumar, M. S. N., Sandell, G., et al. 2006, MNRAS

Dickel, H., Lubenow, A., Goss, W., Forster, J., \& Rots, A. 1983, A\&A, 120, 74
Dickel, J. R., Dickel, H. R., \& Wilson, W. J. 1978, ApJ, 223, 840 Downes, D., \& Rinehart, R. 1966, ApJ, 144, 937

Fixsen, D. J., Bennett, C. L., \& Mather, J. C. 1999, ApJ, 526, 207

Garden, R., Geballe, T. R., Gatley, I., \& Nadeau, D. 1986, MNRAS, 220, 203

Genzel, R., \& Downes, D. 1977, A\&A, 30, 145

Giannini, T., Nisini, B., Lorenzetti, D., et al. 2000, A\&A, 358, 310

Giannini, T., Nisini, B., \& Lorenzetti, D. 2001, ApJ, 555, 40

Goldsmith, P. F., Bergin, E. A., \& Lis, D. C. 1997, ApJ, 491, 615

Graf, U. U., Heyminck, S., Michael, E. A., et al. 2002, in Millimeter and Submillimeter Detectors for Astronomy, ed. T. G. Phillips, \& J. Zmuidzinas, Proc. SPIE, 4855

Habart, E., Abergel, A., Walmsley, C. M., Teyssier, D., \& Pety, J. 2005, A\&A, 437, 177

Hatchell, J., Thompson, M. A., Millar, T. J., \& MacDonald, G. H. 1998, A\&A, 338,713

Jaffe, D., \& Plume, R. 1995, in Airborne Symposium on the Galactic Ecosystem, ed. M. Haas, J. Davidson, \& E. Erickson, ASP Conf. Ser., 73

Jaffe, D. T., Genzel, R., Harris, A. I., et al. 1989, ApJ, 344, 265

Jansen, D. J., van Dishoeck, E. F., Keene, J., Boreiko, R. T., \& Betz, A. L. 1996, A\&A, 309, 899

Jørgensen, J. K., Schöier, F. L., \& van Dishoeck, E. F. 2004, A\&A, 416, 603

Kaufman, M., Wolfire, M., Hollenbach, D., \& Luhman, M. 1999, ApJ, 527, 795

Keene, J., Lis, D., Phillips, T., \& Schilke, P. 1997, in Molecules in Astrophysics: Probes and Processes, ed. E. F. van Dishoeck, IAU Symp., 178 (Dordrecht: Kluwer), 129

Köster, B. 1998, Ph.D. Thesis, Universität zu Köln

Kramer, C., Jakob, H., Mookerjea, B., et al. 2004, A\&A, 424, 887

Kramer, C., Mookerjea, B., Bayet, E., et al. 2005, A\&A, 441, 961

Lane, A., Haas, M., Hollenbach, D., \& Erickson, E. 1990, ApJ, 361, 132

Langer, W. D., \& Penzias, A. A. 1990, ApJ, 357, 477

Leung, H. O., \& Thaddeus, P. 1992, ApJS, 81, 267

Malhotra, S., Kaufman, M., Hollenbach, D., et al. 2001, ApJ, 561, 766

Mangum, J. G., Wootten, A., \& Mundy, L. G. 1992, ApJ, 388, 467

Marston, A. P., Reach, W. T., Noriega-Crespo, A., et al. 2004, ApJS, 154, 333

Mauersberger, R., Wilson, T. L., Walmsley, C. M., Henkel, C., \& Batrla, W. 1985, A\&A, 146, 168

Mookerjea, B., Kramer, C., Röllig, M., \& Masur, M. 2006, A\&A, 456, 235

Motte, F., Bontemps, S., Schilke, P., et al. 2005, in IAU Symp., 151

Oka, T., Iwata, M., Maezawa, H., et al. 2004, ApJ, 602, 803

Ossenkopf, V., Trojan, C., \& Stutzki, J. 2001, A\&A, 378, 608

Palmer, P., Goss, W. M., \& Whiteoak, J. B. 2004, MNRAS, 347, 1164

Poglitsch, A., Herrmann, F., Genzel, R., et al. 1996, ApJ, 462, L43

Pollack, J. B., Hollenbach, D., Beckwith, S., et al. 1994, ApJ, 421, 615

Preibisch, T., Ossenkopf, V., Yorke, H. W., \& Henning, T. 1993, A\&A, 279, 577

Richardson, K. J., Sandell, G., Cunningham, C. T., \& Davies, S. R. 1994, A\&A, 286, 555

Roelfsema, P. R., Goss, W. M., \& Geballe, T. R. 1989, A\&A, 222, 247

Rubin, R. H., Simpson, J. P., Lord, S. D., et al. 1994, ApJ, 420, 772

Schneider, N., Simon, R., Kramer, C., et al. 2003, A\&A, 406, 915

Schneider, N., Bontemps, S., Simon, R., et al. 2006, A\&A, SBS2006

Schroder, K., Staemmler, V., Smith, M. D., Flower, D. R., \& Jaquet, R. 1991, J. Phys. B Atom. Molec. Phys., 24, 2487

Shirley, Y. L., Evans, N. J., Young, K. E., Knez, C., \& Jaffe, D. T. 2003, ApJS, 149, 375

Smith, H. A., Hora, J. L., Marengo, M., \& Pipher, J. L. 2006

Sternberg, A., \& Dalgarno, A. 1989, ApJ, 338, 197

Stutzki, J. 1985, Ph.D. Thesis

Tielens, A., \& Hollenbach, D. 1985, ApJ, 291, 722

Vallée, J. P., \& Fiege, J. D. 2006, ApJ, 636, 332

van Zadelhoff, G.-J., Dullemond, C. P., van der Tak, F. F. S., et al. 2002, A\&A, 395,373

Vastel, C., Spaans, M., Ceccarelli, C., Tielens, A. G. G. M., \& Caux, E. 2001, A\&A, 376, 1064

Walker, C. K., Adams, F. C., \& Lada, C. J. 1990, ApJ, 349, 515

Wendker, H. 1984, A\&AS, 58, 291

Williams, J. A., Dickel, H. R., \& Auer, L. H. 2004, ApJS, 153, 463

Wilson, T., \& Mauersberger, R. 1990, A\&A, 239, 305

Zmuidzinas, J., Betz, A. L., Boreiko, R. T., \& Goldhaber, D. M. 1988, ApJ, 335, 774 


\section{Online Material}




\section{Appendix A: Results at individual positions}

Table 7 lists the derived physical parameters (shell- and modelaveraged) for temperature, $\mathrm{H}_{2}$ number density, volume filling factors, $\mathrm{H}_{2}$ column density, and molecular gas mass. The resulting spectral profiles are compared with the observed lines in Fig. 5. The plots in Fig. 8 show the fit of the integrated emission for $\mathrm{CO},{ }^{13} \mathrm{CO}$, and $\mathrm{C}^{18} \mathrm{O}$ up to $J=20$ in comparison to the observed lines. In the following, we discuss details of the individual positions.

DR21 C: This position covers the DR21 H II-region. Its surrounding molecular cloud (within a radius of $\sim 0.6 \mathrm{pc}$ ) forms the southern part of the molecular ridge. Our best-fit model indicates that the envelope at a temperature of $40 \mathrm{~K}$ comprises roughly $94 \%$ of the total mass, leaving $6 \%$ for the warm (about $150 \mathrm{~K}$ ) and dynamically active gas $\left(v_{\text {turb }}\right.$ between 4.5 and $29 \mathrm{~km} \mathrm{~s}^{-1}$ ) subject to shocks and UV-heating. Jaffe et al. already found indications in 1989 of a warm component with a mass of $55 M_{\odot}(D / 1.7 \mathrm{kpc})^{2}$, which is fully consistent with our result from Table 7 . The by far more massive, colder gas component was only indirectly traced by the CO $J=7-6$ line reversal in their study. In our model, the properties of this gas component in the envelope shell are much better constrained by the $\mathrm{C}^{18} \mathrm{O}$ and ${ }^{13} \mathrm{CO} J=1-0$ transitions, which are close to optically thin $(\tau \sim 0.1$ resp. 0.8$)$. The turnover at $J_{\text {upper }} \sim 12$ (cf. Fig. 8) results from the increasing critical density leading to sub-thermal excitation of the high- $J$ transitions at the clump density of about $3 \times 10^{6} \mathrm{~cm}^{-3}$. Boreiko \& Betz (1991) observed $\mathrm{CO}$ and ${ }^{13} \mathrm{CO} 9-8$ at comparable resolution onboard the Kuiper Airborne Observatory (KAO). We indicate their integrated fluxes in Fig. 8 for DR21 C. There is good agreement with ${ }^{13} \mathrm{CO}$, but CO $J=9-8$ is weaker than the $J=7-6$ line presented here. This may indicate a stronger flattening of the CO rotation curve that could only be explained by a discontinuous density or temperature structure.

In support of a shock driven excitation, Lane et al. (1990) gives an upper limit to CO $J=22-21$ of $2 \times$ $10^{-4} \mathrm{erg} \mathrm{s}^{-1} \mathrm{~cm}^{-2} \mathrm{sr}^{-1}$ in a $44^{\prime \prime} 8$ beam. Considering beam dilution, this limit is still high compared to the intensity of $\mathrm{CO} 17-16$, the highest rotational line considered here. Our prediction with the radiative transfer model of $2 \times$ $10^{-7} \mathrm{erg} \mathrm{s}^{-1} \mathrm{~cm}^{-2} \mathrm{sr}^{-1}$ can explain why this detection attempt failed, although we cannot fully exclude the presence of $>200 \mathrm{~K}$ warm gas.

DR21 E: The east side of the DR21 H II-region is not located on the molecular ridge, but is enriched with gas ejected from the ridge. The lack of cold gas gives rise to an average temperature of $62 \mathrm{~K}$ for a gas mass of $\sim 240 M_{\odot}$. It appears from the Spitzer/IRAC image that a fraction of gas in the east is undergoing a blister phase. Unlike the western outflow side, it is not stopped by a dense cloud and expands unhampered. We find that $20 \%$ of the gas is heated to about $90 \mathrm{~K}$ and accelerated to velocities up to $v=32 \mathrm{~km} \mathrm{~s}^{-1}$. As the radiative transfer code is not designed for outflow geometries, we applied a turbulent velocity gradient from 32 to $5 \mathrm{~km} \mathrm{~s}^{-1}$ to simulate the outflow. For the envelope gas, the asymmetry of the $\mathrm{CO}$ profiles indicates a motion away from the core at $\sim 0.7 \mathrm{~km} \mathrm{~s}^{-1}$. Because of the steep drop in high- $J \mathrm{CO}$ emission, the local clump density was fitted below $n\left(\mathrm{H}_{2}\right)=10^{5} \mathrm{~cm}^{-3}$.
DR21 W: Molecular line emission and radiative transfer models of DR2 $1 \mathrm{~W}$ are similar to position DR21 E: the gas is warmer than in the ridge $(>50 \mathrm{~K})$ but not at densities above $10^{5} \mathrm{~cm}^{-3}$. The warm $(\sim 120 \mathrm{~K})$ gas contributes with a small fraction of about $3.5 \%$ to the total derived mass of $\sim 430 M_{\odot}$. A turbulent velocity gradient between 30 and $11 \mathrm{~km} \mathrm{~s}^{-1}$ is responsible for line wing emission. The $\mathrm{CO}$ lines show only weak self-absorption although the opacity is high at the line centre. This is plausible because the cloud surface is illuminated by the cluster at DR21 C, so we had to implement a positive temperature gradient from $44 \mathrm{~K}$ to $55 \mathrm{~K}$ across the cloud envelope.

DR21 $(\mathrm{OH})$ : The position of DR21 $(\mathrm{OH})$ is dominated by cold gas from the ridge at a temperature below $30 \mathrm{~K}$ over a size of up to $0.7 \mathrm{pc}$. For the modeling, we simplified the scenario of several embedded cores, as reported by, e.g., Mangum et al. (1992) (at possibly different systemic velocities), to a single clumpy core defined by the warm interface region with an outer radius of $0.2 \mathrm{pc}$. The significant mid- and high- $J \mathrm{CO}$ emission is a clear indication of warm and dense gas. We find that only $2 \%$, or $34 M_{\odot}$ of the gas is heated to $\sim 100 \mathrm{~K}$ and at densities of $10^{6} \mathrm{~cm}^{-3}$ or more. There is no evolved outflow associated with this position, but Garden et al. (1986) and Richardson et al. (1994) see indications of a very young outflow associated with high temperatures and shocks. In our model, half of the warm gas mass is at relative velocities $5<v_{\text {turb }}<32 \mathrm{~km} \mathrm{~s}^{-1}$, creating the line wing emission. The observed blue-skewed CO line profiles are compatible with radial infall motion of the envelope gas of $1.0-1.5 \mathrm{~km} \mathrm{~s}^{-1}$.

DR21 FIR1: The northern FIR-region contains at least three sub-sources. Besides FIR1, the $80^{\prime \prime}$ beam partly covers the second source FIR2, so we have to consider this as contamination. The modeled outer radius overlaps slightly with the neighbouring source, but is well within the beam. Because high-J CO is not detected at DR21 FIR1, we can constrain the temperature to $\leq 82 \mathrm{~K}$. Given the CO 7-6 flux at this position, the lower limit of the temperature in the inner region is $T>50 \mathrm{~K}$. The relatively weak self-absorption of the low- $J$ lines is probably due to the presence of moderately warm gas (up to $40 \mathrm{~K}$ ) in the outer surface layers, supporting the flat density profile used in the model.

\section{Appendix B: Correction for reference-beam emission}

Before the implementation of an on-the-fly-mode (OTF), dualbeam-switch (DBS) was the only efficient observing mode to use SMART at KOSMA. Therefore, observations in 2003 and 2004 of CO 7-6 and [C I] had to be performed in DBS-mode. In this mode, a chopping secondary mirror is used for fast periodic switching between an $\mathrm{ON}$ and an OFF position (signaland reference phase). After some time, usually $20 \mathrm{~s}$, determined by the stability of the system, the telescope is moved so that the reference phase is now the ON, and the new OFF position falls exactly on the opposite side. In the data reduction, each OFF position is weighted equally. Slight differences in the optical path lengths usually lead to strong standing waves in the spectra when using a simple BS-mode. The period of these waves corresponds to the optical distance to the subreflector. These standing waves are very efficiently suppressed in DBS-mode. However, mapping the extended sources is rather difficult if the chopper throw (between $\left.5^{\prime}-6^{\prime}\right)$ is small compared to the size of the source and the chopper movement is fixed to one direction (azimuth for 


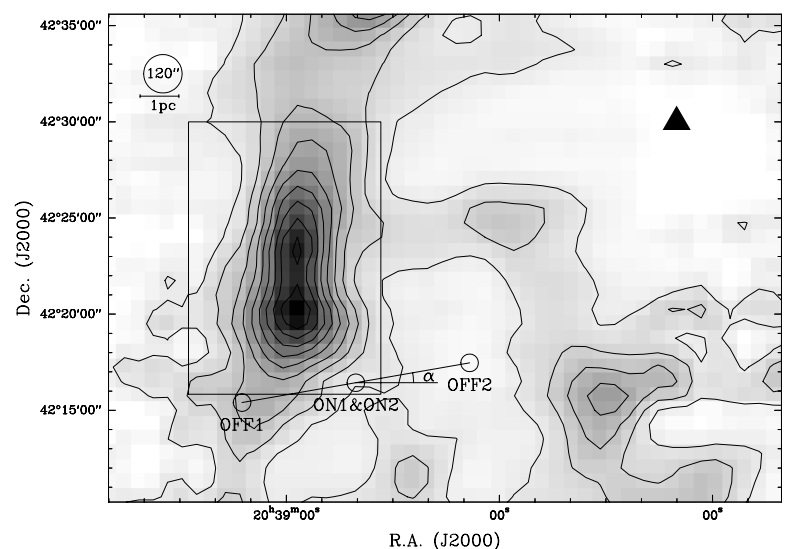

Fig. B.1. OTF map of the ${ }^{13} \mathrm{CO} 2-1$ emission (SBS2006). An emissionfree position is indicated by the triangle on the right and was used as OFF position for the OTF observations. The box indicates the map size of the DBS-mode observations in Figs. 2 and 3. The circle in the top left corner indicates the $120^{\prime \prime}$ beam size. The smaller circles labeled ON1\&ON2, OFF1, and OFF2 show an examplary DBS measurement (cf. Fig. B.2) with a field rotation angle $\alpha=10^{\circ}$.

KOSMA). Under those conditions, mapping needs careful planning to prevent self-chopping within the source. The observing time is thus not only constrained by source visibility but also by sky rotation. For an hour angle of $0^{\mathrm{h}}$, the secondary throw is only in right ascension, while for other angles there is a component in declination. The ${ }^{13} \mathrm{CO} 3-2$ position-switched OTF-map in Fig. B.1 shows that the DR21 ridge is $\mathrm{N}-\mathrm{S}$ orientated and that the field rotation angles $\alpha$ of less than $\pm 30^{\circ}$ are preferred in order to prevent chopping into the ridge of emission with both OFF beams.

To reduce the impact of artefacts remaining after the standard DBS reduction pipeline, all DBS data were corrected in the following way (see Fig. B.2 for an example): (i) regardless of whether the DBS-spectra (Fig. B.2a) show self-chopping or not, the emission in the difference (Fig. B.2b) of the two OFF positions is analysed. Any significant signal here is considered as pollution. This assumption holds as long as at least one of the two OFF positions is free of emission and no absorption against the continuum is present. In the example (Figs. B.2c and d) only the OFF-position of Beam 1 (OFF1) is contaminated; (ii) if a signal in the difference is detected above the noise level, the algorithm tries to reconstruct the emission signal (Fig. B.2d) by removing Beam 1 from the final spectrum (Fig. B.2e), i.e. we use only ON2-OFF2 here. This scheme works well for situations where standing waves are negligible. In cases where standingwave patterns are persistent, we transform the spectra to Fourierspace, mask-out the standing wave frequencies, and transform the data back to user-space. Due to the shorter integration time, this procedure increases the noise level by a factor of $\sqrt{2}$. In case of DR21, this method successfully recovered the emission, because one OFF position was always nearly free of emission. If, however, the source extends over more than twice the chop throw in all directions, this method does not work.

\section{Appendix C: LTE methods}

A simple determination of the excitation temperature and the column density of C I can be made if we assume the emission is optically thin and the level popolation is given by thermal

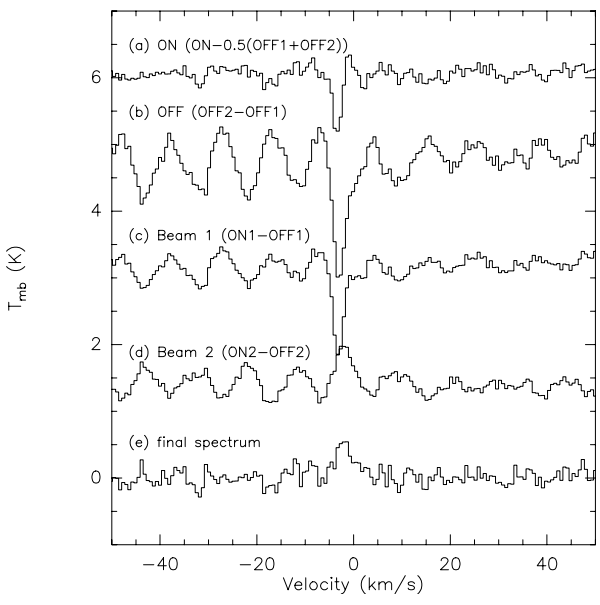

Fig. B.2. The method of correcting self-chopping in dual-beamswitch observing mode. The uppermost spectrum a) shows a standard DBS spectrum of CI 1-0 with a self-chopped absorption line $(\mathrm{ON}-0.5(\mathrm{OFF} 1+\mathrm{OFF} 2))$, and b) shows the difference signal (OFF2-OFF1). In the middle, $\mathbf{c}$ ) and d), both beams of the DBS spectrum are displayed (Beam 1 or ON1-OFF1, and resp. for Beam 2). The result e) (after removing standing waves) shows a faint emission line.

excitation. The column density of a material in an excited state $u$ is given by:

$N_{u}=\frac{8 \pi k_{\mathrm{B}} v^{2}}{h c^{3} A_{u}} \int \frac{T_{\mathrm{mb}} \mathrm{d} v}{\mathrm{Kkm} \mathrm{s}^{-1}}$

With Einstein A coefficients for atomic carbon $A_{1}=7.9 \times$ $10^{-8} \mathrm{~s}^{-1}$ and $A_{2}=2.7 \times 10^{-7} \mathrm{~s}^{-1}$ (Schroder et al. 1991), the column densities for each transition are

$N_{1}=5.96 \times 10^{15} \int \frac{T_{\mathrm{mb}}\left(\mathrm{C} \mathrm{I}^{3} \mathrm{P}_{1}-{ }^{3} \mathrm{P}_{0}\right) \mathrm{d} v}{\mathrm{~K} \mathrm{~km} \mathrm{~s}^{-1}}\left[\mathrm{~cm}^{-2}\right]$

and

$N_{2}=4.72 \times 10^{15} \int \frac{T_{\mathrm{mb}}\left(\mathrm{C} \mathrm{I}^{3} \mathrm{P}_{2}-{ }^{3} \mathrm{P}_{1}\right) \mathrm{d} v}{\mathrm{~K} \mathrm{~km} \mathrm{~s}^{-1}}\left[\mathrm{~cm}^{-2}\right]$.

To derive the exitation temperature, we assume LTE and substitute $N_{1}$ and $N_{2}$ using Boltzmann statistics:

$$
\begin{gathered}
\frac{N_{\mathrm{u}}}{N_{\mathrm{l}}}=\frac{g_{\mathrm{u}}}{g_{1}} \mathrm{e}^{-\frac{\left(E_{\mathrm{u}}-E_{1}\right) / k_{\mathrm{B}}}{T_{\mathrm{ex}}}} \\
T_{\mathrm{ex}}=\frac{\frac{h v_{2}}{k_{\mathrm{B}}}}{\ln \left(\frac{N_{1}}{N_{2}} \frac{g_{2}}{g_{1}}\right)}=\frac{38.8 \mathrm{~K}}{\ln \left(\frac{1.26}{R_{\mathrm{Cl}}} \frac{5}{3}\right)}=\frac{38.8 \mathrm{~K}}{\ln \left(2.11 / R_{\mathrm{CI}}\right)},
\end{gathered}
$$

where $R_{\mathrm{C}_{\mathrm{I}}}$ is the ratio of integrated intensities (defined in (C.2) and (C.3)), and the statistical weight is $g_{J}=2 J+1$. Although the temperature of the 3-level state of [C I] is very sensitive to small variations in $R_{\mathrm{CI}}$, the total column density depends only weakly on the temperature:

$$
\begin{aligned}
N(\mathrm{C} \mathrm{I})= & \sum_{J=0}^{2} \mathrm{~N}_{J}=N_{1} \frac{Z}{3 \mathrm{e}^{-\frac{23.6 \mathrm{~K}}{T_{\mathrm{ex}}}}} \\
= & 5.96 \times 10^{15} \frac{1+3 \mathrm{e}^{\frac{-23.6 \mathrm{~K}}{T_{\mathrm{ex}}}}+5 \mathrm{e}^{\frac{-62.4 \mathrm{~K}}{T_{\mathrm{ex}}}}}{3 \mathrm{e}^{\frac{-23.6 \mathrm{~K}}{T_{\mathrm{ex}}}}} \\
& \times \int \frac{T_{\mathrm{mb}}\left(\mathrm{C} \mathrm{I}^{3} \mathrm{P}_{1}-{ }^{3} \mathrm{P}_{0}\right) \mathrm{d} v}{\mathrm{~K} \mathrm{~km} \mathrm{~s}^{-1}}\left[\mathrm{~cm}^{-2}\right]
\end{aligned}
$$

where $Z=\sum_{J=0}^{2} \mathrm{~g}_{J} \mathrm{e}^{-\frac{E_{J}}{k_{\mathrm{B}} T_{\mathrm{ex}}}}$ is the partition function. 
To estimate the excitation temperature from the two transitions ${ }^{13} \mathrm{CO} J=3-2$ and $J=1-0$, we use Eq. (C.4) which then translates into

$T_{\text {ex,low }}=-\frac{\left(E_{3}-E_{1}\right) / k_{\mathrm{B}}}{\ln \left(\frac{N_{3}}{N_{1}} \frac{g_{1}}{g_{3}}\right)}=\frac{26.4 \mathrm{~K}}{\ln \left(9 / R_{13} \mathrm{CO}, \text { low }\right)}$.

The transition $J=6-5$ and $J=3-2$ accordingly gives

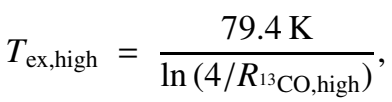

where $R_{{ }^{13} \mathrm{CO} \text {,low }}$ (resp. $R_{{ }^{13} \mathrm{CO} \text {,high }}$ ) is the integrated intensity ratio of 3-2/1-0 (resp. 6-5/3-2).

The ${ }^{13} \mathrm{CO}$ and $\mathrm{C}^{18} \mathrm{O}$ column density can then be approximated by these equations: $\begin{aligned} N\left({ }^{13} \mathrm{CO}\right)= & 1.268 \times 10^{12}\left(T_{\mathrm{ex}}+0.88 \mathrm{~K}\right) \mathrm{e}^{111.1 \mathrm{~K} / T_{\mathrm{ex}}} \\ & \times \int \frac{T_{\mathrm{mb}}\left({ }^{13} \mathrm{CO}(6-5)\right) \mathrm{d} v}{\mathrm{~K} \mathrm{~km} \mathrm{~s}}\left[\mathrm{~cm}^{-1}\right],\end{aligned}$

$$
N\left({ }^{13} \mathrm{CO}\right)=4.71 \times 10^{13}\left(T_{\mathrm{ex}}+0.88 \mathrm{~K}\right) \mathrm{e}^{5.27 \mathrm{~K} / T_{\mathrm{ex}}}
$$$$
\times \int \frac{T_{\mathrm{mb}}\left({ }^{13} \mathrm{CO}(1-0)\right) \mathrm{d} v}{\mathrm{~K} \mathrm{~km} \mathrm{~s}^{-1}}\left[\mathrm{~cm}^{-2}\right]
$$

$$
N\left(\mathrm{C}^{18} \mathrm{O}\right)=4.60 \times 10^{13}\left(T_{\mathrm{ex}}+0.88 \mathrm{~K}\right) \mathrm{e}^{5.27 \mathrm{~K} / T_{\text {ex }}}
$$

$$
\times \int \frac{T_{\mathrm{mb}}\left(\mathrm{C}^{18} \mathrm{O}(1-0)\right) \mathrm{d} v}{\mathrm{~K} \mathrm{~km} \mathrm{~s}^{-1}}\left[\mathrm{~cm}^{-2}\right]
$$

Using Eq. (C.4), an upper limit to the excitation temperature of $\mathrm{O}$ I can be derived from the [O I] 145/63 $\mu \mathrm{m}$ ratio (fluxes in $\operatorname{erg~s}{ }^{-1} \mathrm{~cm}^{-2} \mathrm{sr}^{-1}$ ) by:

$T_{\mathrm{ex}}=\frac{227.7 \mathrm{~K}}{\ln \left(2.83 /\left(12.22 \cdot R_{[\mathrm{OI}]}\right)\right)}$. 